\title{
THE TRACE ON PROJECTIVE REPRESENTATIONS OF QUANTUM GROUPS
}

\author{
NATHAN GEER AND BERTRAND PATUREAU-MIRAND
}

\begin{abstract}
For certain roots of unity, we consider the categories of weight modules over three quantum groups: small, un-restricted and unrolled. The first main theorem of this paper is to show that there is a modified trace on the projective modules of the first two categories. The second main theorem is to show that category over the unrolled quantum group is ribbon. Partial results related to these theorems were known previously.
\end{abstract}

\section{INTRODUCTION}

For an odd ordered root of unity $\xi$ and lattice $L$, let $\mathcal{U}_{\xi}^{L}, \mathcal{U}_{\xi}^{H}$ and $\overline{\mathcal{U}}_{\xi}^{L}$ be the unrestricted, unrolled and small quantum groups, respectively (see Section 3). Let $\mathscr{C}_{\text {odd }}$ (resp. $\mathscr{C}_{\text {odd }}^{H}$, resp. $\overline{\mathscr{C}}_{\text {odd }}$ ) be the category of $\mathcal{U}_{\xi}^{L}$ (resp. $\mathcal{U}^{H}$, resp. $\overline{\mathcal{U}}_{\xi}^{L}$ ) weight modules. The usual construction of quantum invariants do not directly apply to these categories because of the following obstructions: the categories are not semi-simple and have vanishing quantum dimensions. Partial results overcoming these obstructions have been obtained in $[9,17,18,19,20,22$. In this paper we generalize some of these results using a new concept called generically semi-simple (loosely meaning the category is graded and semi-simple on a dense portion of the graded pieces). In [3, 6], the results of this paper will be used in future work to construct topological invariants.

The first part of this paper contains a general theory with two main theorems which extend properties of generic simple objects to general properties in the full category. The first (Theorem 9) loosely says that if a category is generically semi-simple, pivotal and braided such that there is a twist for every generic simple object then the full category has a twist and so the category is ribbon. The second (Theorem 14) loosely says that if a category is generically semi-simple and pivotal with a right trace on its projective objects whose modified dimension satisfies $\mathrm{d}(V)=\mathrm{d}\left(V^{*}\right)$ for all generic simple objects $V$ then the right trace is a (two sided) trace.

We apply these theorems to the categories of modules over the different quantum groups mentioned above. It was previously known that $\mathscr{C}_{\text {odd }}^{H}$ is a braided pivotal category and $\mathscr{C}_{\text {odd }}$ is a pivotal category with a right trace, see [19] and [18], respectively. Here we remark that $\mathscr{C}_{\text {odd }}^{H}$ and $\mathscr{C}_{\text {odd }}$ are generically semi-simple (see Subsection 4.3). In Subsection 4.4 we show that each generic simple module in $\mathscr{C}_{\text {odd }}^{H}$ has a twist and thus Theorem 9 implies $\mathscr{C}_{\text {odd }}^{H}$ is ribbon. Subsection 4.5 contains a proof that the modified dimension satisfies $\mathrm{d}(V)=\mathrm{d}\left(V^{*}\right)$ for all generic simple objects $V$ of $\mathscr{C}_{\text {odd }}$ and so Theorem 14 implies the unique right trace (up to global scalar) on the ideal of projective modules of $\mathscr{C}_{\text {odd }}$ is a trace. This unique trace (up to global scalar) induces a trace on $\overline{\mathscr{C}}_{\text {odd }}$.

The main theorems in this paper about $\mathscr{C}_{\text {odd }}^{H}$ and $\mathscr{C}_{\text {odd }}$ use deep results developed by De Concini, Kac, Procesi, Reshetikhin and Rosso in the series of papers [10, 11, 12, 13. In particular, we use the quantum co-adjoint action. In general, these results hold for odd ordered roots of unity.

Date: October 31, 2016. 
However, in Section 5 we show directly that in the case of $\mathfrak{s l}(2)$ the results discussed above hold for even and odd ordered roots of unity. In particular, we show $\mathscr{C}_{\mathfrak{s l}(2)}$ is braided and $\mathscr{C}_{\mathfrak{s l}(2)}^{H}$ and $\overline{\mathscr{C}}_{\mathfrak{s l}(2)}$ have unique traces. Finally, in Section 6 we conjecture that Subsections 4.4 and 4.5 generalize to even ordered root of unity.

\section{TENSOR CATEgORIES}

In this section we give the basic definitions for tensor categories and the functors induced by graphic calculus.

1.1. Pivotal and ribbon categories. We recall the definition of pivotal and ribbon tensor categories, for more details see for instance, [1. In this paper, we consider strict tensor categories with tensor product $\otimes$ and unit object $\mathbb{I}$. Let $\mathscr{C}$ be such a category. The notation $V \in \mathscr{C}$ means that $V$ is an object of $\mathscr{C}$.

The category $\mathscr{C}$ is a pivotal category if it has duality morphisms

$$
\overrightarrow{\operatorname{coev}}_{V}: \mathbb{I} \rightarrow V \otimes V^{*}, \quad \overrightarrow{\mathrm{ev}}_{V}: \quad V^{*} \otimes V \rightarrow \mathbb{I}, \quad \overleftarrow{\operatorname{coev}}_{V}: \mathbb{I} \rightarrow V^{*} \otimes V \quad \text { and } \quad \overleftarrow{\mathrm{ev}}_{V}: \quad V \otimes V^{*} \rightarrow \mathbb{I}
$$

which satisfy compatibility conditions (see for example [1, 18]). In particular, the left dual and right dual of a morphism $f: V \rightarrow W$ in $\mathscr{C}$ coincide:

$$
\begin{aligned}
f^{*} & =\left(\overrightarrow{\mathrm{ev}}_{W} \otimes \operatorname{Id}_{V^{*}}\right)\left(\operatorname{Id}_{W^{*}} \otimes f \otimes \operatorname{Id}_{V^{*}}\right)\left(\operatorname{Id}_{W^{*}} \otimes \overrightarrow{\operatorname{coev}}_{V}\right) \\
& =\left(\operatorname{Id}_{V^{*}} \otimes \overleftarrow{\mathrm{ev}}_{W}\right)\left(\operatorname{Id}_{V^{*}} \otimes f \otimes \operatorname{Id}_{W^{*}}\right)\left(\overleftarrow{\operatorname{coev}}_{V} \otimes \operatorname{Id}_{W^{*}}\right): W^{*} \rightarrow V^{*}
\end{aligned}
$$

Then there is a natural notion of right categorical (partial) trace in $\mathscr{C}$ : for any $V, W \in \mathscr{C}$,

$$
\begin{aligned}
\operatorname{tr}_{R}: \operatorname{End}_{\mathscr{C}}(V) & \rightarrow \mathbb{k} \\
f & \mapsto \overleftarrow{\mathrm{ev}}_{V}\left(f \otimes \operatorname{Id}_{V^{*}}\right) \overrightarrow{\operatorname{cov}}_{V} \text { and } \\
\operatorname{ptr}_{R}: \operatorname{End}_{\mathscr{C}}(V \otimes W) & \rightarrow \operatorname{End}_{\mathscr{C}}(V) \\
f & \mapsto\left(\operatorname{Id}_{V} \otimes \overleftarrow{\mathrm{ev}}_{W}\right)\left(f \otimes \operatorname{Id}_{W^{*}}\right)\left(\operatorname{Id}_{V} \otimes \overrightarrow{\operatorname{cov}}_{W}\right)
\end{aligned}
$$

and analogous left categorical (partial) trace $\operatorname{tr}_{L}$ (resp. $\operatorname{ptr}_{L}$ ) defined using $\overrightarrow{\mathrm{ev}}$ and $\overleftarrow{c o e v}$.

A braiding on $\mathscr{C}$ consists of a family of natural isomorphisms $\left\{c_{V, W}: V \otimes W \rightarrow W \otimes V\right\}$ satisfying the Hexagon Axiom:

$$
c_{U, V \otimes W}=\left(\operatorname{Id}_{V} \otimes c_{U, W}\right) \circ\left(c_{U, V} \otimes \operatorname{Id}_{W}\right) \quad \text { and } \quad c_{U \otimes V, W}=\left(c_{U, W} \otimes \operatorname{Id}_{V}\right) \circ\left(\operatorname{Id}_{U} \otimes c_{V, W}\right)
$$

for all $U, V, W \in \mathscr{C}$. We say $\mathscr{C}$ is braided if it has a braiding. If $\mathscr{C}$ is pivotal and braided, one can define a family of natural automorphisms

$$
\theta_{V}=\operatorname{ptr}_{R}\left(c_{V, V}\right): V \rightarrow V .
$$

We say that $\mathscr{C}$ is ribbon and the morphism $\theta$ is a twist if

$$
\theta_{V^{*}}=\left(\theta_{V}\right)^{*}
$$

for all $V \in \mathscr{C}$.

Remark 1. An equivalent definition of a ribbon category is a braided left rigid balanced category. Left rigid means that there are left duals $\left(V, V^{*}, \overrightarrow{\mathrm{ev}}_{V}, \overrightarrow{\mathrm{cev}}_{V}\right)$ and the balance $\theta$ is a natural automorphism of the identity functor satisfying $\theta_{U \otimes V}=\left(\theta_{U} \otimes \theta_{V}\right) \circ c_{V \otimes U} \circ c_{U \otimes V}$ and Equation (11). 
1.2. $\mathbb{k}$-categories. Let $\mathbb{k}$ be a integral domain. A $\mathbb{k}$-category is a category $\mathscr{C}$ such that its homsets are left $\mathbb{k}$-modules, the composition of morphisms is $\mathbb{k}$-bilinear, and the canonical $\mathbb{k}$-algebra $\operatorname{map}_{\mathbb{k}} \rightarrow \operatorname{End}_{\mathscr{C}}(\mathbb{I}), k \mapsto k \operatorname{Id}_{\mathbb{I}}$ is an isomorphism. A tensor $\mathbb{k}$-category is a tensor category $\mathscr{C}$ such that $\mathscr{C}$ is a $\mathbb{k}$-category and the tensor product of morphisms is $\mathbb{k}$-bilinear. An object $V$ of $\mathscr{C}$ is simple if $\operatorname{End}_{\mathscr{C}}(V)=\mathbb{k} \operatorname{Id}_{V}$. Let $V$ be an object in $\mathscr{C}$ and let $\alpha: V \rightarrow W$ and $\beta: W \rightarrow V$ be morphisms. The triple $(V, \alpha, \beta)$ (or just the object $V$ ) is a retract of $W$ if $\beta \alpha=\operatorname{Id}_{V}$. An object $W$ is a direct sum of the finite family $\left(V_{i}\right)_{i}$ of objects of $\mathscr{C}$ if there exist retracts $\left(V_{i}, \alpha_{i}, \beta_{i}\right)$ of $W$ with $\beta_{i} \alpha_{j}=0$ for $i \neq j$ and $\operatorname{Id}_{W}=\sum_{i} \alpha_{i} \beta_{i}$. An object which is a direct sum of simple objects is called semi-simple.

1.3. Traces on ideals in pivotal categories. Here we recall the definition of a (right) trace on an (right) ideal in a pivotal $\mathbb{k}$-category $\mathscr{C}$, for more details see [22]. By a right ideal of $\mathscr{C}$ we mean a full subcategory $\mathcal{I}$ of $\mathscr{C}$ such that:

(1) If $V \in \mathcal{I}$ and $W \in \mathscr{C}$, then $V \otimes W \in \mathcal{I}$.

(2) If $V \in \mathcal{I}$ and if $W \in \mathscr{C}$ is a retract of $V$, then $W \in \mathcal{I}$.

One defines similarly the notion of a left ideal by replacing in the above definition $V \otimes W \in \mathcal{I}$ by $W \otimes V \in \mathcal{I}$. A full subcategory $\mathcal{I}$ of $\mathscr{C}$ is an ideal if it is both a right and left ideal.

If $\mathcal{I}$ is a right ideal in $\mathscr{C}$ then a right trace on $\mathcal{I}$ is a family of linear functions

$$
\left\{\mathrm{t}_{V}: \operatorname{End}_{\mathscr{C}}(V) \rightarrow \mathbb{k}\right\}_{V \in \mathcal{I}}
$$

such that following two conditions hold:

(1) If $U, V \in \mathcal{I}$ then for any morphisms $f: V \rightarrow U$ and $g: U \rightarrow V$ in $\mathscr{C}$ we have

$$
\mathrm{t}_{V}(g f)=\mathrm{t}_{U}(f g) \text {. }
$$

(2) If $U \in \mathcal{I}$ and $W \in \mathscr{C}$ then for any $f \in \operatorname{End}_{\mathscr{C}}(U \otimes W)$ we have

$$
\mathrm{t}_{U \otimes W}(f)=\mathrm{t}_{U}\left(\operatorname{ptr}_{R}(f)\right)
$$

The notion of a left trace on a left ideal is obtained by replacing (2) in the above definition with $\mathrm{t}_{W \otimes U}(f)=\mathrm{t}_{U}\left(\operatorname{ptr}_{L}(f)\right)$ for all $f \in \operatorname{End}_{\mathscr{C}}(W \otimes U)$. A family $\mathrm{t}=\left\{\mathrm{t}_{V}\right\}_{V \in \mathcal{I}}$ is a trace if $\mathcal{I}$ is an ideal and $\mathrm{t}$ is both a left and right trace.

The class of projective modules Proj in $\mathscr{C}$ is an ideal. In a pivotal category projective and injective objects coincide (see [22]). The ideal Proj is an important example which we will consider later in this paper.

1.4. Colored ribbon graph invariants. Let $\mathscr{C}$ be a pivotal category. A morphism $f: V_{1} \otimes$ $\cdots \otimes V_{n} \rightarrow W_{1} \otimes \cdots \otimes W_{m}$ in $\mathscr{C}$ can be represented by a box and arrows:

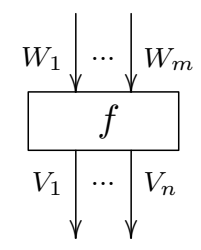

Boxes as above are called coupons. By a ribbon graph in an oriented manifold $\Sigma$, we mean an oriented compact surface embedded in $\Sigma$ which decomposed into elementary pieces: bands, annuli, and coupons (see [27]) and is the thickening of an oriented graph. In particular, the vertices of 
the graph lying in Int $\Sigma=\Sigma \backslash \partial \Sigma$ are thickened to coupons. A $\mathscr{C}$-colored ribbon graph is a ribbon graph whose (thickened) edges are colored by objects of $\mathscr{C}$ and whose coupons are colored by morphisms of $\mathscr{C}$. The intersection of a $\mathscr{C}$-colored ribbon graph in $\Sigma$ with $\partial \Sigma$ is required to be empty or to consist only of vertices of valency 1 . When $\Sigma$ is a surface the ribbon graph is just a tubular neighborhood of the graph.

A $\mathscr{C}$-colored ribbon graph in $\mathbb{R}^{2}$ is called planar. A $\mathscr{C}$-colored ribbon graph in $S^{2}=\mathbb{R}^{2} \cup\{\infty\}$ is called spherical. A $\mathscr{C}$-colored ribbon graph in $\mathbb{R}^{3}$ or $\mathbb{R}^{2} \times[0,1]$ are called spatial.

For $i \in\{2,3\}$, the $\mathscr{C}$-colored ribbon graphs in $\mathbb{R}^{i-1} \times[0,1]$ form a category $\operatorname{Gr}_{\mathscr{C}}^{i}$ as follows: objects of $\operatorname{Gr}_{\mathscr{C}}^{i}$ are finite sequences of pairs $(X, \varepsilon)$, where $X \in \mathscr{C}$ and $\varepsilon= \pm$. Morphisms of $\operatorname{Gr}_{\mathscr{C}}^{i}$ are isotopy classes of $\mathscr{C}$-colored ribbon graphs in $\mathbb{R}^{i-1} \times[0,1]$. By a (1,1)-ribbon graph in $\operatorname{Gr}_{\mathscr{C}}^{i}$ we mean a $\mathscr{C}$-colored ribbon graph which is an endomorphism of an object $(V,+)$ in $\operatorname{Gr}_{\mathscr{C}}^{i}$. Let $\mathrm{F}: \mathrm{Gr}_{\mathscr{C}}^{i} \rightarrow \mathscr{C}$ be the Reshetikhin-Turaev $\mathbb{k}$-linear functor (see [21]). If $\mathscr{C}$ is a ribbon category, the functor on planer graphs $\mathrm{F}: \mathrm{Gr}_{\mathscr{C}}^{2} \rightarrow \mathscr{C}$ extends to the functor on spatial graphs $\mathrm{F}: \mathrm{Gr}_{\mathscr{C}}^{3} \rightarrow \mathscr{C}$.

1.5. Renormalized colored ribbon graph invariants. The motivation of this paper is to provide the underpinnings for the construction of topological invariants. With this in mind, in this subsection, we recall the notion of re-normalized colored ribbon graph invariants introduced and studied in [19, 17, 20, 21, 22]. This subsection is independent of the rest of the paper. The theory of re-normalized invariants produces non-trivial invariants in some situations when the standard approaches fail. In particular, these invariants can be non-trivial when quantum dimension vanish. The re-normalized invariant of closed $\mathscr{C}$-colored ribbon graph can be computed in three steps: 1) cut a special edge of a closed $\mathscr{C}$-colored ribbon graph, 2) apply $\mathrm{F}$ to the resulting graph to obtain an endomorphism and 3) apply to the endomorphism a $\mathbb{k}$-linear functional to obtain a number. If this functional has certain properties then the number is an invariant of the ribbon graph. Here is a more precise definition:

Let $\mathscr{C}$ be a pivotal (resp., ribbon) category. Let $\mathcal{T}_{a d m}$ be a class of planar (resp. spatial) $\mathscr{C}$-colored (1,1)-ribbon graphs. We denote an element of $\mathcal{T}_{a d m}$ by $T_{V}$ where $V$ is the object of $\mathscr{C}$ which colors the open edge of the (1,1)-ribbon graph. We call $V$ the section of $T_{V}$. Given such a graph $T_{V}$ the right braid closure gives a well defined equivalence class of a closed ribbon graph $\widehat{T_{V}}$ in $\mathbb{R}^{2}$ (resp. in $\mathbb{R}^{3}$ ). Let $\mathcal{L}_{a d m}$ be the class of right braid closures of elements of $\mathcal{T}_{a d m}$. Let $\mathrm{t}=\left\{\mathrm{t}_{V}: \operatorname{End}_{\mathscr{C}}(V) \rightarrow \mathbb{k}\right\}_{V}$ be a family of linear maps where $V$ runs over all the sections of elements $T_{V} \in \mathcal{T}_{a d m}$. Suppose that $\mathrm{t}$ satisfies the condition:

If $T_{V}, T_{W}^{\prime} \in \mathcal{T}_{a d m}$ such that $\widehat{T_{V}}$ is isotopic to $\widehat{T_{W}^{\prime}}$ then $\mathrm{t}_{V}\left(\mathrm{~F}\left(T_{V}\right)\right)=\mathrm{t}_{W}\left(\mathrm{~F}\left(T_{W}^{\prime}\right)\right)$.

We call the function

$$
\mathrm{F}^{\prime}: \mathcal{L}_{a d m} \rightarrow \mathbb{k} \text { defined by } \mathrm{F}^{\prime}\left(\widehat{T_{V}}\right)=\mathrm{t}_{V}\left(\mathrm{~F}\left(T_{V}\right)\right)
$$

the re-normalized invariant associated to $\mathcal{L}_{a d m}$ and t.

We will now give some examples of re-normalized invariants.

Example 1: $T$-ambi pair. Let A be a class of simple objects in a pivotal $\mathbb{k}$-category $\mathscr{C}$. The classes $\mathcal{T}_{a d m}$ and $\mathcal{L}_{a d m}$ are formed by the trivalent ribbon planar graphs whose edges are colored by elements of $\mathrm{A}$. The family $\mathrm{t}=\left(\mathrm{t}_{V}\right)_{V \in \mathrm{A}}$ is determined by a mapping $\mathrm{d}: \mathrm{A} \rightarrow \mathbb{k}^{\times}$constant on isomorphism classes of objects. Then the map $\mathrm{t}_{V}$ is determined by $\mathrm{t}_{V}\left(\lambda \operatorname{Id}_{V}\right)=\lambda \mathrm{d}(V)$. If $\mathrm{F}^{\prime}$ is invariant by isotopy in the sphere $S^{2}$ (here we consider an isomorphism $S^{2} \simeq \mathbb{R}^{2} \cup\{\infty\}$ ) then we say that (A,d) is a trivalent ambidextrous pair or $t$-ambi for short. A (modified) $6 j$-symbol is the value of a tetrahedron under $\mathrm{F}^{\prime}$. These 
$6 j$-symbols are the elementary algebraic ingredients of a re-normalized Turaev-Viro type invariant of 3-manifolds defined by state sums on triangulations ([21, 19]).

Example 2: Right trace. Let $\mathrm{t}$ be a right trace on a right ideal $\mathcal{I}$ in a pivotal $\mathbb{k}$-category $\mathscr{C}$, see Subsection 1.3. Let $\mathcal{T}_{a d m}$ be all the $\mathscr{C}$-colored $(1,1)$-ribbon planar graphs whose sections are in $\mathcal{I}$. Then $\mathrm{F}^{\prime}$ is a invariant of planar isotopy but in general it is not an invariant of isotopy in the sphere $S^{2}$. Nevertheless, for $V \in \mathcal{I}$ one can set $\mathrm{d}(V)=\mathrm{t}_{V}\left(\operatorname{Id}_{V}\right)$ then Corollary 7 of [22] implies that for $\mathrm{A}=\left\{V \in \mathcal{I}: V\right.$ is simple, $\left.V^{*} \in \mathcal{I}, \mathrm{d}(V)=\mathrm{d}\left(V^{*}\right)\right\}$, $(A, \mathrm{~d})$ is a trivalent-ambidextrous pair and the restriction of $\mathrm{F}^{\prime}$ to $\mathrm{A}$-colored graphs is an invariant of isotopy in the sphere $S^{2}$.

Example 3: (Two-sided) trace. Let $\mathscr{C}$ be a pivotal (resp. ribbon) $\mathbb{k}$-category and let $\mathrm{t}$ be a trace on an ideal $\mathcal{I}$. Again, let $\mathcal{T}_{a d m}$ be all $\mathscr{C}$-colored $(1,1)$-ribbon planar (resp. spatial) graphs whose sections are in $\mathcal{I}$. By setting $\mathrm{d}(V)=\mathrm{t}_{V}\left(\operatorname{Id}_{V}\right)$ for $V \in \mathcal{I}$ then Theorem 5 of 22] implies $\mathrm{F}^{\prime}$ is an invariant of spherical (resp. spatial) ribbon graphs. Moreover, $\mathcal{L}_{a d m}$ is formed by the $\mathscr{C}$-colored ribbon graphs with at least one edge in $\mathcal{I}$.

\section{6. $\mathcal{G}$-graded and generically $\mathcal{G}$-semi-simple categories. We now fix a group $\mathcal{G}$.}

Definition 2. Grading:

A pivotal $\mathbb{k}$-category is $\mathcal{G}$-graded if for each $g \in \mathcal{G}$ we have a non-empty full subcategory $\mathscr{C}_{g}$ of $\mathscr{C}$ stable by retract such that

(1) $\mathscr{C}=\bigoplus_{g \in \mathcal{G}} \mathscr{C}_{g}$,

(2) if $V \in \mathscr{C}_{g}$, then $V^{*} \in \mathscr{C}_{g^{-1}}$,

(3) if $V \in \mathscr{C}_{g}, V^{\prime} \in \mathscr{C}_{g^{\prime}}$ then $V \otimes V^{\prime} \in \mathscr{C}_{g g^{\prime}}$,

(4) if $V \in \mathscr{C}_{g}, V^{\prime} \in \mathscr{C}_{g^{\prime}}$ and $\operatorname{Hom}_{\mathscr{C}}\left(V, V^{\prime}\right) \neq 0$, then $g=g^{\prime}$.

For a subset $\mathcal{X} \subset \mathcal{G}$ we say:

(1) $\mathcal{X}$ is symmetric if $\mathcal{X}^{-1}=\mathcal{X}$

(2) $\mathcal{X}$ is small in $\mathcal{G}$ if the group $\mathcal{G}$ can not be covered by a finite number of translated copies of $\mathcal{X}$, in other words, for any $g_{1}, \ldots, g_{n} \in \mathcal{G}$, we have $\bigcup_{i=1}^{n}\left(g_{i} \mathcal{X}\right) \neq \mathcal{G}$.

Definition 3. Semi-simplicity:

(1) $A \mathbb{k}$-category $\mathscr{C}$ is semi-simple if all its objects are semi-simple.

(2) $A \mathbb{k}$-category $\mathscr{C}$ is finitely semi-simple if it is semi-simple and has finitely many isomorphism classes of simple objects.

(3) A $\mathcal{G}$-graded category $\mathscr{C}$ is a generically $\mathcal{G}$-semi-simple category (resp. generically finitely $\mathcal{G}$-semi-simple category) if there exists a small symmetric subset $\mathcal{X} \subset \mathcal{G}$ such that for each $g \in \mathcal{G} \backslash \mathcal{X}, \mathscr{C}_{g}$ is semi-simple (resp. finitely semi-simple). We call $\mathcal{X}$ the singular locus of $\mathscr{C}$. By a generic simple object we mean a simple object of $\mathscr{C}_{g}$ for some $g \in \mathcal{G} \backslash \mathcal{X}$.

Remark 4. For a generically $\mathcal{G}$-semi-simple category $\mathscr{C}$ with singular locus $\mathcal{X}$, its ideal Proj of projective objects contains all objects of $\mathscr{C}_{g}$ for $g \in \mathcal{G} \backslash \mathcal{X}$. In particular, generic simple objects of $\mathscr{C}$ are projective.

The notion of generically $\mathcal{G}$-semi-simple categories appears in [21, 19] through the following generalization of fusion categories (in particular, fusion categories satisfy the following definition when $\mathcal{G}$ is the trivial group, $\mathcal{X}=\emptyset$ and $\mathrm{d}=\mathrm{b}=\operatorname{qdim}_{\mathscr{C}}$ is the quantum dimension): 
Definition 5 (Relative $\mathcal{G}$-spherical category). Let $\mathscr{C}$ be a generically finitely $\mathcal{G}$-semi-simple pivotal $\mathbb{k}$-category with singular locus $\mathcal{X} \subset \mathcal{G}$ and let $\mathrm{A}$ be the class of generic simple objects of $\mathscr{C}$. We say that $\mathscr{C}$ is $(\mathcal{X}, \mathrm{d})$-relative $\mathcal{G}$-spherical if

(1) there exists a map $\mathrm{d}: \mathrm{A} \rightarrow \mathbb{k}^{\times}$such that $(\mathrm{A}, \mathrm{d})$ is a t-ambi pair,

(2) there exists a map $\mathrm{b}: \mathrm{A} \rightarrow \mathbb{k}$ such that $\mathrm{b}(V)=\mathrm{b}\left(V^{*}\right), \mathrm{b}(V)=\mathrm{b}\left(V^{\prime}\right)$ for any isomorphic objects $V, V^{\prime} \in \mathrm{A}$ and for any $g_{1}, g_{2}, g_{1} g_{2} \in \mathcal{G} \backslash \mathcal{X}$ and $V \in \mathcal{G}_{g_{1} g_{2}}$ we have

$$
\mathrm{b}(V)=\sum_{V_{1} \in \operatorname{irr}\left(\mathscr{C}_{g_{1}}\right), V_{2} \in \operatorname{irr}\left(\mathscr{C}_{g_{2}}\right)} \mathrm{b}\left(V_{1}\right) \mathrm{b}\left(V_{2}\right) \operatorname{dim}_{\mathbb{k}}\left(\operatorname{Hom}_{\mathscr{C}}\left(V, V_{1} \otimes V_{2}\right)\right)
$$

where $\operatorname{irr}\left(\mathscr{C}_{g_{i}}\right)$ denotes a representing set of isomorphism classes of simple objects of $\mathscr{C}_{g_{i}}$.

We finish this section by recalling the following theorem and corollary which we use later to show certain algebras are semi-simple. For a proof of the theorem see [14].

Theorem 6 (the Density Theorem). Let $A$ be an algebra over an algebraically closed field $k$. Let $\left\{V_{i}\right\}_{i}$ be a set of irreducible pairwise nonisomorphic finite dimensional modules over $A$. Then the map $\oplus_{i} \rho_{i}: A \rightarrow \bigoplus_{i} \operatorname{End}_{k}\left(V_{i}\right)$ is surjective.

Corollary 7. Let $\mathscr{C}$ be the category of finite dimensional modules of the finite dimensional algebra A. Let $\left\{V_{i}\right\}_{i}$ be a set of irreducible pairwise non-isomorphic finite dimensional modules over $A$. If

$$
\operatorname{dim}(A)=\sum_{i} \operatorname{dim}\left(V_{i}\right)^{2}
$$

then $\mathscr{C}$ is semi-simple.

Proof. From the Density Theorem we know that $\oplus_{i} \rho_{i}: A \rightarrow \bigoplus_{i} \operatorname{End}_{k}\left(V_{i}\right)$ is surjective. The assumption on the dimensions implies that this map is a injective. Thus, $A$ is isomorphic to the direct sum of matrix algebras which is semi-simple and it follows that $\mathscr{C}$ is semi-simple.

\section{ExTENSION OF GENERIC PROPERTIES}

Let $\mathscr{C}$ be a pivotal $\mathbb{k}$-category. In this section we present two theorems which extend properties observed for generic simple objects of $\mathscr{C}$ to general properties in the full category.

Proposition 8. If $\mathscr{C}$ is a braided pivotal $\mathbb{k}$-category then the class of objects

$$
\left\{V \in \mathscr{C}: \theta_{V^{*}}=\left(\theta_{V}\right)^{*}\right\}
$$

forms a full subcategory of $\mathscr{C}$ which is ribbon.

Proof. For each object $V \in \mathscr{C}$, consider the automorphism

$$
\mathscr{E}_{V}=\operatorname{ptr}_{R}\left(c_{V, V}^{-1}\right) \circ \operatorname{ptr}_{R}\left(c_{V, V}\right)=\mathrm{F}\left(\begin{array}{l}
\bigcirc \\
\bigcirc
\end{array}\right) \in \operatorname{End}_{\mathscr{C}}(V)
$$

The family $\left(\mathscr{E}_{V}\right)_{V \in \mathscr{C}}$ defines a natural transformation (as $\mathscr{E}_{*}$ is an automorphism of the identity functor, its naturality is just the fact that $\mathscr{E}_{V} f=f \mathscr{E}_{U}$ for any $f: U \rightarrow V$ ). Its inverse is given by

$$
\mathscr{E}_{V}^{-1}=\mathrm{F}(\mathcal{O})=\operatorname{ptr}_{L}\left(c_{V, V}^{-1}\right) \circ \operatorname{ptr}_{L}\left(c_{V, V}\right)=\operatorname{ptr}_{L}\left(c_{V, V}\right) \circ \operatorname{ptr}_{L}\left(c_{V, V}^{-1}\right) \text {. }
$$


The naturality of $c_{V, V}$ implies that the image by $\mathrm{F}$ of a $\mathscr{C}$-colored diagram is invariant by Reidemester II and III moves so that for any $V, W \in \mathscr{C}$

$$
\mathscr{E}_{V \otimes W}=\mathrm{F}\left(\begin{array}{c}
\bigcirc \\
\hdashline
\end{array}\right)=\mathrm{F}\left(10 \mathscr{E}_{V} \otimes \mathscr{E}_{W},\right.
$$

showing that $\mathscr{E}_{*}$ is a monoidal transformation. Finally, the dual of the right partial trace is given by the left partial trace so

$$
\left(\mathscr{E}_{V}\right)^{*}=\mathscr{E}_{V^{*}}^{-1}
$$

For any object $V \in \mathscr{C},\left(\theta_{V}\right)^{*}=\left(\mathscr{E}_{V}\right)^{*} \circ \theta_{V^{*}}$ so $\left(\theta_{V}\right)^{*}=\theta_{V^{*}} \Longleftrightarrow \mathscr{E}_{V}=\operatorname{Id}_{V}$ and the class in the proposition is clearly a sub-pivotal category of $\mathscr{C}$ because of properties (2), (3) of the natural automorphism $\mathscr{E}_{*}$.

The following theorem generalizes an argument of Ha (see [23, Proposition 3.7]).

Theorem 9. Let $\mathscr{C}$ be a generically $\mathcal{G}$-semi-simple pivotal braided category. If $\theta(V)^{*}=\theta\left(V^{*}\right)$ holds for any generic simple object $V$, then $\mathscr{C}$ is a ribbon category i.e. $\theta$ is a twist on the full category $\mathscr{C}$.

Proof. Consider the natural automorphism $\mathscr{E}_{*}$ of the proof of Proposition 8 . By assumption we have $\mathscr{E}_{V}=\mathrm{Id}_{V}$ for any generic simple object $V$. By naturality, this property is stable by direct sums so it is also true for any semi-simple object that is a direct sum of generic simple objects. Now for any homogenous object $W \in \mathscr{C}_{g}$, let $h \in \mathcal{G}$ be such that $h, h g \notin \mathcal{X}$ and let $V \in \mathscr{C}_{h}$. Then $V \otimes W \in \mathscr{C}_{h g}$ is semi-simple and

$$
\operatorname{Id}_{V} \otimes \operatorname{Id}_{W}=\mathscr{E}_{V \otimes W}=\mathscr{E}_{V} \otimes \mathscr{E}_{W}=\operatorname{Id}_{V} \otimes \mathscr{E}_{W}
$$

thus $\mathscr{E}_{W}=\operatorname{Id}_{W}$ and $\theta(W)^{*}=\theta\left(W^{*}\right)$.

For the second theorem we first recall the relation between (right) trace and duality:

Proposition 10. (see [22, Lemma 2 \& 3])

(1) If $\mathcal{I}$ is a right ideal then $\mathcal{I}^{*}=\left\{V \in \mathscr{C}, V^{*} \in \mathcal{I}\right\}$ is a left ideal and

$$
\mathcal{I} \text { is an ideal } \Longleftrightarrow \mathcal{I}^{*}=\mathcal{I} \text {. }
$$

(2) If $\mathrm{t}$ is a right trace on the right ideal $\mathcal{I}$ then $\mathrm{t}^{*}$ is a left trace on $\mathcal{I}^{*}$ where by definition, $\mathrm{t}_{V}^{*}(f)=\mathrm{t}_{V^{*}}\left(f^{*}\right)$ for any $V \in \mathcal{I}$, any $f \in \operatorname{End}_{\mathscr{C}}(V)$ and

$$
\mathrm{t} \text { is a trace } \Longleftrightarrow \mathrm{t}^{*}=\mathrm{t} \text {. }
$$

\section{Definition 11.}

(1) A pseudo-monoid class in a pivotal category $\mathscr{C}$ is a class of objects stable by dual, retracts and tensor product.

(2) Let $\mathrm{t}$ be a right trace on a right ideal $\mathcal{I}$. The horizontal part of $\mathcal{I}$ for $\mathrm{t}$ is the class

$$
\mathcal{I}^{=}=\left\{V \in \mathcal{I}: V^{*} \in \mathcal{I} \text { and } \mathrm{t}_{V}=\mathrm{t}_{V}^{*}\right\}
$$

Remark 12. An ideal of a pivotal category is a pseudo-monoid and the converse is partially true. Indeed one could define the idealizer of a pseudo-monoid $\mathcal{M}$ to be the full subcategory of $\mathscr{C}$ whose objects are

$$
\mathbb{I}_{\mathscr{C}}(\mathcal{M})=\{V \in \mathscr{C}: V \otimes \mathcal{M} \subset \mathcal{M} \supset \mathcal{M} \otimes V\}
$$


Then one can prove that $\mathbb{I}_{\mathscr{C}}(\mathcal{M})$ is a sub-pivotal category of $\mathscr{C}$ which contains $\mathcal{M}$ such that $\mathcal{M}$ is an ideal in $\mathbb{I}_{\mathscr{C}}(\mathcal{M})$.

Proposition 13. Let $\mathrm{t}$ be a right trace on a right ideal $\mathcal{I}$. Then the horizontal part $\mathcal{I}=$ of $\mathcal{I}$ is a pseudo-monoid. Furthermore, $\mathcal{I}^{=}$is stable by direct sum in the following weak sense: if $\left\{V_{i}\right\}_{i}$ is a finite family of object of $\mathcal{I}^{=}$and $W \in \mathcal{I} \cap \mathcal{I}^{*}$ is isomorphic to their direct sum, then $W \in \mathcal{I}^{=}$.

Proof. If $V$ is a retract of $W \in \mathcal{I}^{=}$then there are $\alpha: V \rightarrow W, \beta: W \rightarrow V$ with $\beta \alpha=\operatorname{Id}_{V}$. Then $V^{*}$ is a retract of $W^{*} \in \mathcal{I}^{=}$so $V^{*} \in \mathcal{I}$. For any $f \in \operatorname{End}_{\mathscr{C}}(V)$, by definition $\mathrm{t}_{V}^{*}(f)=\mathrm{t}_{V^{*}}\left(f^{*}\right)$ but

$$
\mathrm{t}_{V}(f)=\mathrm{t}_{V}(f \beta \alpha)=\mathrm{t}_{W}(\alpha f \beta)=\mathrm{t}_{W^{*}}\left(\beta^{*} f^{*} \alpha^{*}\right)=\mathrm{t}_{V^{*}}\left(\alpha^{*} \beta^{*} f^{*}\right)=\mathrm{t}_{V^{*}}\left(f^{*}\right) .
$$

So $V \in \mathcal{I}^{=}$and $\mathcal{I}^{=}$is stable by retract (recall in particular that an isomorphic object is a retract).

Let $V \in \mathcal{I}^{=}$and let $\phi_{V}: V \stackrel{\sim}{\rightarrow} V^{* *}$ be the pivotal isomorphism. Then for any $f \in \operatorname{End}_{\mathscr{C}}(V)$, $\mathrm{t}_{V}(f)=\mathrm{t}_{V}\left(\phi_{V}^{-1} f^{* *} \phi_{V}\right)=\mathrm{t}_{V^{* *}}\left(f^{* *} \phi_{V} \phi_{V}^{-1}\right)=\mathrm{t}_{V^{* *}}\left(f^{* *}\right)$. Given $g \in \operatorname{End}_{\mathscr{C}}\left(V^{*}\right)$ then $g$ is the dual of $f=\phi_{V} g^{*} \phi_{V}^{-1} \in \operatorname{End}_{\mathscr{C}}(V)$ so

$$
\mathrm{t}_{V^{*}}(g)=\mathrm{t}_{V^{*}}\left(f^{*}\right)=\mathrm{t}_{V}(f)=\mathrm{t}_{V^{* *}}\left(f^{* *}\right)=\mathrm{t}_{V^{*}}^{*}(g)
$$

and $\left(\mathcal{I}^{=}\right)^{*} \subset \mathcal{I}^{=}$.

Let $V, W \in \mathcal{I}^{=}$and let $f \in \operatorname{End}_{\mathscr{C}}(V \otimes W)$. Then $V^{*} \in \mathcal{I}$ and $\mathrm{t}$ is right ambidextrous by [22, Lemma 4] so $\mathrm{t}\left(\operatorname{ptr}_{R}(f)^{*}\right)=\mathrm{t}\left(\operatorname{ptr}_{L}\left(f^{\prime}\right)\right)=\mathrm{t}\left(\operatorname{ptr}_{L}(f)\right)$ where $f^{\prime}=\left(\phi_{V} \otimes \operatorname{Id}_{W}\right) f\left(\phi_{V}^{-1} \otimes \operatorname{Id}_{W}\right)$ so

$$
\mathrm{t}(f)=\mathrm{t}\left(\operatorname{ptr}_{R}(f)\right)=\mathrm{t}\left(\operatorname{ptr}_{R}(f)^{*}\right)=\mathrm{t}\left(\operatorname{ptr}_{L}(f)\right)=\mathrm{t}\left(\operatorname{ptr}_{L}(f)^{*}\right)=\mathrm{t}\left(\operatorname{ptr}_{R}\left(f^{*}\right)\right)=\mathrm{t}\left(f^{*}\right)
$$

thus $V \otimes W \in \mathcal{I}^{=}$.

If $W$ is a direct sum of objects of $\mathcal{I}^{=}$with associated projectors $p_{i}=\alpha_{i} \beta_{i}$, then so is $W^{*}$ with projectors $p_{i}^{*}$. Now for any $f \in \operatorname{End}_{\mathscr{C}}(W), f=\left(\sum_{i} p_{i}\right) f\left(\sum_{i} p_{i}\right)=\sum_{i, j} p_{i} f p_{j}$ so

$$
\mathrm{t}(f)=\sum_{i} \mathrm{t}\left(p_{i} f p_{i}\right)+\sum_{i \neq j} \mathrm{t}\left(f p_{j} p_{i}\right)=\sum_{i} \mathrm{t}\left(\beta_{i} f \alpha_{i} \beta_{i} \alpha_{i}\right)=\sum_{i} \mathrm{t}\left(\alpha_{i}^{*} f^{*} \beta_{i}^{*}\right)=\mathrm{t}\left(f^{*}\right) .
$$

An object $V \in \mathscr{C}$ is non-zero if $\operatorname{Id}_{V} \neq 0$. For the next theorem, we will do the following assumption that is true for example if $\mathscr{C}$ is a category of finite dimensional representations of a Hopf $\mathbb{k}$-algebra: We will assume that $\mathscr{C}$ is a Krull-Schmidt category and that for any nonzero $V \in \mathscr{C}, V \otimes \cdot$ and $\cdot \otimes V$ are faithful functors. The former implies that any object in $\mathscr{C}$ is isomorphic to an unique direct sum of indecomposable objects (an object is indecomposable if it is not isomorphic to a direct sum of two non-zero objects).

Theorem 14. Let $\mathscr{C}$ be a generically $\mathcal{G}$-semi-simple pivotal category with the above assumption. Let $\mathrm{t}$ be a right trace on Proj and let $\mathrm{d}=\{\mathrm{d}(V) \in \mathbb{k}\}_{V \in \operatorname{Proj}}$ denote the associated modified dimension. If $\mathrm{d}(V)=\mathrm{d}\left(V^{*}\right)$ holds for any generic simple object $V$, then $\mathrm{t}$ is a trace on Proj.

Proof. By assumption we have that any generic simple object $V$ is in $\operatorname{Proj}^{=}$as $\operatorname{End}_{\mathscr{C}}(V) \simeq \mathbb{k} \simeq$ $\operatorname{End}_{\mathscr{C}}\left(V^{*}\right)$ so the right trace on these endomorphisms is determined by $\mathrm{d}(V)=\mathrm{d}\left(V^{*}\right)$. Since Proj $^{=}$is stable by direct sums it contains any semi-simple object that is a direct sum of generic simple objects. Let $W$ be a indecomposable projective object. Then there exists $g$ such that $W \in \mathscr{C}_{g}$. Let $h \in \mathcal{G}$ be such that $h, h^{-1} g \notin \mathcal{X}$ (recall that $\mathcal{X}$ is small) and let $V \in \mathscr{C}_{h}$. Then $W \in \mathcal{I}_{V}=$ Proj thus there exists $U \in \mathscr{C}$ such that $W$ is a retract of $V \otimes U$. As $\mathscr{C}=\bigoplus_{g \in \mathcal{G}} \mathscr{C}_{g}$, we can assume up to replacing $U$ by one of its summand that $U \in \mathscr{C}_{h^{-1}}$. Then $U$ is a semi-simple direct sum of generic simple objects and an element of $\operatorname{Proj}^{=}$. Since $\mathrm{Proj}^{=}$is a pseudo-monoid 
then $V \otimes U \in \operatorname{Proj}^{=}$and $W \in \operatorname{Proj}^{=}$as $W$ is a retract. In addition, since Proj $=$ is stable by direct sums we get that $W \in \mathrm{Proj}^{=}$even if $W \in$ Proj is not indecomposable. Thus, Proj= $=$ Proj and $\mathrm{t}=\mathrm{t}^{*}$ on Proj. In other words, $\mathrm{t}$ is a trace.

\section{QuAntum groups at RoOts of Unity}

In this section we first recall some of the deep results established by De Concini, Kac, Procesi, Reshetikhin and Rosso in the series of papers [10,,11], 112] and [13]. Then we observe that the twist $\theta$ and the modified dimension $\mathrm{d}$ are generically self dual. As a consequence we get the existence of a ribbon structure associated to the unrolled quantum group and the existence of a trace on Proj for the categories of weight modules over the small, unrolled and unrestricted groups.

3.1. The small, the unrolled and the unrestricted. Let $\mathfrak{g}$ be a simple finite-dimensional complex Lie algebra of rank $n$ and dimension $2 N+n$ with the following:

(1) a Cartan subalgebra $\mathfrak{h}$,

(2) a root system consisting in simple roots $\left\{\alpha_{1}, \ldots, \alpha_{n}\right\} \subset \mathfrak{h}^{*}$,

(3) a Cartan matrix $A=\left(a_{i j}\right)_{1 \leq i, j \leq n}$,

(4) a set $\Delta^{+}$of $N$ positive roots,

(5) a root lattice $L_{R}=\bigoplus_{i} \mathbb{Z} \alpha_{i} \subset \mathfrak{h}^{*}$,

(6) a scalar product $\langle\cdot, \cdot\rangle$ on the real span of $L_{R}$ given by its matrix $D A=\left(\left\langle\alpha_{i}, \alpha_{j}\right\rangle\right)_{i j}$ where $D=\operatorname{diag}\left(d_{1}, \ldots, d_{n}\right)$ and the minimum of all the $d_{i}$ is 1 .

The Cartan subalgebra has a basis $\left\{H_{i}\right\}_{i=1 \cdots n}$ determined by $\alpha_{i}\left(H_{j}\right)=a_{j i}$ and its dual basis of $\mathfrak{h}^{*}$ is the fundamental weights basis which generate the lattice of weights $L_{W}$. Let $\rho=\frac{1}{2} \sum_{\alpha \in \Delta^{+}} \alpha \in$ $L_{W}$.

Let $q$ be an indeterminate and for $i=1, \ldots, n$, let $q_{i}=q^{d_{i}}$. Let $\ell$ be an integer such that $\ell \geq 2$ (and $\ell \notin 3 \mathbb{Z}$ if $\mathfrak{g}=G_{2}$ ). Let $\xi=\mathrm{e}^{2 \sqrt{-1} \pi / \ell}$ and $r=\frac{2 \ell}{3+(-1)^{\ell}}$. For $x \in \mathbb{C}$ and $k, l \in \mathbb{N}$ we use the notation:

$$
\xi^{x}=\mathrm{e}^{\frac{2 i \pi x}{\ell}}, \quad\{x\}_{q}=q^{x}-q^{-x}, \quad[x]_{q}=\frac{\{x\}_{q}}{\{1\}_{q}}, \quad[k]_{q} !=[1]_{q}[2]_{q} \cdots[k]_{q}, \quad\left[\begin{array}{c}
k \\
l
\end{array}\right]_{q}=\frac{[k]_{q} !}{[l]_{q} ![k-l]_{q} !} .
$$

Let $\mathbb{K}_{\ell}$ be the subring of $\mathbb{C}(q)$ made of fraction that have no poles at $\xi$ ( $\mathbb{K}_{\ell}$ is a localization of $\mathbb{C}[q]$ ). A $\mathbb{K}_{\ell}$-module can be specialized at $q=\xi$ (the specialization is the tensor product with the

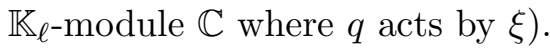

For each lattice $L$ with $L_{R} \subset L \subset L_{W}$, there is an associated quantum group which contains the

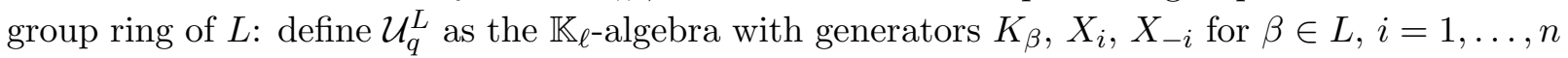
and relations

$$
\begin{gathered}
K_{0}=1, \quad K_{\beta} K_{\gamma}=K_{\beta+\gamma}, \quad K_{\beta} X_{\sigma i} K_{-\beta}=q^{\sigma\left\langle\beta, \alpha_{i}\right\rangle} X_{\sigma i}, \\
{\left[X_{i}, X_{-j}\right]=\delta_{i j} \frac{K_{\alpha_{i}}-K_{\alpha_{i}}^{-1}}{q_{i}-q_{i}^{-1}},} \\
\sum_{k=0}^{1-a_{i j}}(-1)^{k}\left[\begin{array}{c}
1-a_{i j} \\
k
\end{array}\right]_{q_{i}} X_{\sigma i}^{k} X_{\sigma j} X_{\sigma i}^{1-a_{i j}-k}=0, \text { if } i \neq j \\
9
\end{gathered}
$$


where $\sigma= \pm 1$. Drinfeld and Jimbo consider the quantum group corresponding to $L_{R}$. The algebra $\mathcal{U}_{q}^{L}$ is a Hopf algebra with coproduct $\Delta$, counit $\epsilon$ and antipode $S$ defined by

$$
\begin{aligned}
& \Delta\left(X_{i}\right)=1 \otimes X_{i}+X_{i} \otimes K_{\alpha_{i}}, \quad \Delta\left(X_{-i}\right)=K_{\alpha_{i}}^{-1} \otimes X_{-i}+X_{-i} \otimes 1, \\
& \Delta\left(K_{\beta}\right)=K_{\beta} \otimes K_{\beta}, \quad \epsilon\left(X_{i}\right)=\epsilon\left(X_{-i}\right)=0, \quad \epsilon\left(K_{\alpha_{i}}\right)=1, \\
& S\left(X_{i}\right)=-X_{i} K_{\alpha_{i}}^{-1}, \quad S\left(X_{-i}\right)=-K_{\alpha_{i}} X_{-i}, \quad S\left(K_{\beta}\right)=K_{-\beta} \text {. }
\end{aligned}
$$

The unrestricted quantum group $\mathcal{U}_{\xi}^{L}$ is the $\mathbb{C}$-algebra obtained from $\mathcal{U}_{q}^{L}$ by specializing $q$ to $\xi$.

The unrolled quantum group $\mathcal{U}^{H}=\mathcal{U}_{\xi}^{H}$ is the algebra generated by $K_{\beta}, X_{i}, X_{-i}, H_{i}$ for $\beta \in$ $L, i=1, \ldots, n$ with Relations (4),(5), (6) where $q=\xi$ plus the relations

$$
\left[H_{i}, X_{\sigma j}\right]=\sigma a_{i j} X_{\sigma j}, \quad\left[H_{i}, H_{j}\right]=\left[H_{i}, K_{\beta}\right]=0
$$

where $\sigma= \pm 1$. The algebra $\mathcal{U}^{H}$ is a Hopf algebra with coproduct $\Delta$, counit $\epsilon$ and antipode $S$ defined as above on $K_{\beta}, X_{i}, X_{-i}$ and defined on the elements $H_{i}$ for $i=1, \ldots, n$ by

$$
\Delta\left(H_{i}\right)=1 \otimes H_{i}+H_{i} \otimes 1, \quad \epsilon\left(H_{i}\right)=0, \quad S\left(H_{i}\right)=-H_{i} .
$$

For a fixed choice of a convex order $\beta_{*}=\left(\beta_{1}, \ldots, \beta_{N}\right)$ of $\Delta^{+}$define recursively a convex set of root vectors $\left(X_{ \pm \beta}\right)_{\beta \in \beta_{*}}$ in $\mathcal{U}_{\xi}^{L}$ (see Section 8.1 and 9.1 of [7]). The small quantum group $\overline{\mathcal{U}}_{\xi}^{L}$ is the quotient of $\mathcal{U}_{\xi}^{L}$ by the relations

$$
X_{ \pm \beta}^{r}=0 \text { and } K_{\gamma}^{2 r}=1 \text { for all } \beta \in \Delta_{+}, \gamma \in L_{R}
$$

The dimension of $\overline{\mathcal{U}}_{\xi}^{L}$ is $r^{2 N}(2 r)^{n}\left[L: L_{R}\right]$. From now on if it is clear we will not write the superscript $L$ in $\mathcal{U}_{\xi}^{L}$ or $\overline{\mathcal{U}}_{\xi}^{L}$

3.2. Pivotal structures. The element $\phi=K_{2 \rho}^{1-r}$ in $U$ where $U$ is $\mathcal{U}_{\xi}, \mathcal{U}^{H}$ or $\overline{\mathcal{U}}_{\xi}$ satisfies $S^{2}(x)=$ $\phi x \phi^{-1}$. It follows that $U$ is a pivotal Hopf algebra and the category of finite dimensional $U$ modules is a pivotal $\mathbb{C}$-categories, for details see [4, 19]. Here the dual is given by the dual vector space $V^{*}=\operatorname{Hom}_{\mathbb{C}}(V, \mathbb{C})$ equipped with the transpose action composed with antipodal morphism $S$. The duality morphisms $\overrightarrow{\mathrm{ev}}$ and $\overrightarrow{\mathrm{coev}}$ are the standard evaluation and coevaluation whereas $\overleftarrow{\mathrm{ev}}=\overrightarrow{\mathrm{ev}} \circ \tau \circ(\phi \otimes 1)$ and $\overleftarrow{\mathrm{oov}}=\tau \circ\left(\phi^{-1} \otimes 1\right) \circ \overrightarrow{\mathrm{coev}}$ where $\tau$ is the flip.

Remark 15. The $h$-adic version of the quantum group $U_{h}(\mathfrak{g})=U_{h}$ is the $\mathbb{C}[[h]]$-topological Hopf algebra with generators $X_{i}, X_{-i}, H_{i}$ for $i=1, \ldots, n$ and Relations (5), (6) and (7) where $q$ and $K_{\alpha_{i}}$ are replaced by $\mathrm{e}^{h / 2}$ and $q_{i}^{H_{i}}$, respectively. The category of finite dimensional topologically free $U_{h}$-modules is a pivotal category where the pivotal element is normally give by $K_{2 \rho}$. In this paper we use $\phi=K_{2 \rho}^{1-r}$ instead of $K_{2 \rho}$ which also defines a pivotal structure. The reason we make this choice is because the pivotal structure for $\phi=K_{2 \rho}^{1-r}$ is compatible with the braiding and trace consider in future subsections. The pivotal structure corresponding to $K_{2 \rho}$ does not have such compatibilities: the left and right twist differ by a square and the modified dimensions of $V$ and $V^{*}$ are not equal.

\section{Quantum GRoups AT ODD ORDERED RoOts OF UNITY}

In this section we consider the case when $\ell$ is an odd integer. In this case, $\ell=r$. To make the notation clear we will write $\mathscr{C}_{\text {odd }}, \mathscr{C}_{\text {odd }}^{H}$ and $\overline{\mathscr{C}}_{\text {odd }}$ for the categories in this section. 
4.1. Weight modules. Let $Z_{0}$ be the subalgebra of $\mathcal{U}_{\xi}^{L}$ generated by $\left\{X_{ \pm \beta}^{r}, K_{\gamma}^{r}: \beta \in \Delta_{+}, \gamma \in\right.$ $L\}$. Then $Z_{0}$ is a sub-Hopf algebra contained in the center of $\mathcal{U}_{\xi}^{L}$. Moreover, $Z_{0}$ is isomorphic to the Hopf algebra of regular functions of a group $\mathcal{G}=\operatorname{Spec}\left(Z_{0}\right)$ which is Poisson-Lie dual to a complex simple Lie group with Lie algebra $\mathfrak{g}$ (see [11]). Here $\operatorname{Spec}\left(Z_{0}\right)$ is the algebraic variety of algebra homomorphisms from $Z_{0}$ to $\mathbb{C}$.

By a $\mathcal{U}_{\xi}$-weight module we mean a finite dimensional module over $\mathcal{U}$ which restrict to a semisimple module over $Z_{0}$. Since $X_{ \pm \beta}^{r}=0$ and $K_{\gamma}^{2 r}=1$ in $\overline{\mathcal{U}}_{\xi}$ we say all finite dimensional $\overline{\mathcal{U}}_{\xi^{-}}$ modules are weight modules. Finally, a finite dimensional $\mathcal{U}^{H}$-module $V$ is a $\mathcal{U}^{H}$-weight module if it is a semi-simple module over the subalgebra generated by $\left\{H_{i}: i=1 \cdots n\right\}$ and for any $\gamma=\sum_{i} c_{i} \alpha_{i} \in L$ then $q^{\sum_{i} d_{i} c_{i} H_{i}}=K_{\gamma}$ as operators on $V$. Let $\mathscr{C}_{\text {odd }}$ (resp. $\mathscr{C}_{\text {odd }}^{H}$, resp. $\overline{\mathscr{C}}_{\text {odd }}$ ) be the category of $\mathcal{U}_{\xi}$ (resp. $\mathcal{U}^{H}$, resp. $\overline{\mathcal{U}}_{\xi}$ ) weight modules. As the action of each $K_{\beta}$ is determined by the action of the $H_{i}$ 's it follows that $\mathscr{C}_{\text {odd }}^{H}$ does not depend on the lattice $L$.

For $g \in \mathcal{G}=\operatorname{Hom}_{\text {alg }}\left(Z_{0}, \mathbb{C}\right)$ let $\mathscr{C}_{g}$ be the full subcategory of $\mathscr{C}_{\text {odd }}$ whose objects are modules where each $z \in Z_{0}$ act by $g(z)$. For $g \in \mathcal{G}$ with $g\left(X_{ \pm \beta}^{r}\right)=0$ for all $\beta \in \Delta_{+}$we say $g$ is diagonal and the modules of $\mathscr{C}_{g}$ are nilpotent. Similarly, $\mathscr{C}_{\text {odd }}^{H}$ is graded by the group $\mathcal{D}$ of diagonal elements of $\mathcal{G}$.

The dual of a weight module is a weight module. This implies that the category $\mathscr{C}_{\text {odd }}$ (resp. $\mathscr{C}_{\text {odd }}^{H}$, resp. $\left.\overline{\mathscr{C}}_{\text {odd }}\right)$ is a sub-pivotal $\mathbb{C}$-category of the category of all finite dimensional modules.

4.2. The braiding on $\mathscr{C}_{\text {odd }}^{H}$. In this subsection we briefly recall the existence of a braiding for $\mathscr{C}_{\text {odd }}^{H}$. We refer to Theorem 41 of [19] for details.

Recall the $h$-adic quantum group $U_{h}(\mathfrak{g})=U_{h}$ defined in Remark 15. For a root $\beta \in L_{R}$, let $q_{\beta}=q^{\langle\beta, \beta\rangle / 2}$. Let $\exp _{q}(x)=\sum_{i=0}^{\infty} \frac{(1-q)^{i} x^{i}}{\left(1-q^{i}\right)\left(1-q^{i-1}\right) \cdots(1-q)}$. Consider the following elements of the $h$-adic version of the quantum group $U_{h}$ :

$$
H H^{h}=q^{\sum_{i, j} d_{i}\left(A^{-1}\right)_{i j} H_{i} \otimes H_{j}}, \quad \check{R}^{h}=\prod_{\beta \in \beta_{*}} \exp _{q_{\beta}^{-2}}\left(\left(q_{\beta}-q_{\beta}^{-1}\right) X_{\beta} \otimes X_{-\beta}\right)
$$

where the product is ordered by the convex order $\beta_{*}$ of $\Delta^{+}$. It is well known that $R^{h}=H H^{h} \check{R}^{h}$ defines a quasi-triangular structure on $U_{h}$ (see for example [24]).

Let $\exp _{q}^{<}(x)=\sum_{i=0}^{r-1} \frac{(1-q)^{i} x^{i}}{\left(1-q^{i}\right)\left(1-q^{i-1}\right) \cdots(1-q)}$. Replacing the occurrences of $\exp _{q_{\beta}^{-2}}$ with $\exp _{q_{\beta}^{-2}}^{<}$in the formula of $\check{R}^{h}$ gives an element of $\mathcal{U}_{q} \otimes \mathcal{U}_{q}$ (seen as a sub-algebra of $U_{h}\left[h^{-1}\right]^{\otimes 2}$ ) called the truncated quasi-R-matrix. Specializing for $q=\xi$ we get an element $\check{R}^{<} \in \mathcal{U}_{\xi} \otimes \mathcal{U}_{\xi}$. The action of $R^{<}=H H^{h} \check{R}^{<}$determines a braiding on $\mathscr{C}^{H}$ given by $c_{V, W}(v \otimes w)=\tau\left(R^{<}(v \otimes w)\right)$ where $\tau$ is the flip. Summarizing we have:

Proposition 16. The category $\mathscr{C}_{\text {odd }}^{H}$ is a braided pivotal category.

4.3. Semi-simple. Here we explain why $\mathscr{C}_{\text {odd }}$ and $\mathscr{C}_{\text {odd }}^{H}$ are generically semi-simple categories. The $g$-th graded piece of $\mathscr{C}_{\text {odd }}$ can be described using ideals of the algebra: $\mathscr{C}_{g}$ is the category of weight module whose annihilator contains the two sided ideal $I_{g}$ generated by $\left\{z-g(z): z \in Z_{0}\right\}$. Hence $\mathscr{C}_{g}$ is identified with the category of finite dimensional modules of the finite dimensional algebra $\mathcal{U}_{\xi} / I_{g}$. For generic $g$, we will prove that the algebra $\mathcal{U}_{\xi} / I_{g}$ is semi-simple which will imply the category $\mathscr{C}_{g}$ is semi-simple.

A diagonal element of $g \in \mathcal{G}$ is regular if $g\left(K_{ \pm \beta}^{r}\right) \neq \pm 1$ for all $\beta \in \Delta_{+}$. For regular $g$, the category $\mathscr{C}_{g}$ has $r^{n}$ non-isomorphic highest weight irreducible modules $V_{i}$. These modules are also modules over $\mathcal{U}_{\xi}^{L_{R}}$ which is a subset of $\mathcal{U}_{\xi}^{L}$. Then Corollary 3.2 of [10] implies that these 
modules have dimension $r^{N}$. Therefore, we have $\sum_{i} \operatorname{dim}\left(V_{i}\right)^{2}=r^{2 N+n}$. The PBW theorem implies $\operatorname{dim}\left(\mathcal{U}_{\xi}^{L}\right)=\sum_{i} \operatorname{dim}\left(V_{i}\right)^{2}$. Thus, Corollary 7 implies that for regular $g$, the category $\mathscr{C}_{g}$ semi-simple.

De Concini and Kac consider certain derivations $\underline{e}_{i}$ and $\underline{f}_{i}$ for $i=1, \ldots, n$. For each $t \in \mathbb{C}$, in [10, Section 3.5], it is shown these derivations give automorphisms exp $t \underline{e}_{i}$ and $\exp t \underline{f}_{i}$ of (a completion of) the algebra $\mathcal{U}_{\xi}$. Let $\widetilde{G}$ be the group generated by all these automorphisms. This group leaves $Z_{0}$ invariant and acts as holomorphic transformations on the algebraic variety $\operatorname{Spec}\left(Z_{0}\right)$. The action of $\widetilde{G}$ on $\operatorname{Spec}\left(Z_{0}\right)$ is called the quantum coadjoint action. Theorem 6.1 of [1] considers the orbits of this action. In particular, part (d) of this theorem says that the union of all $\widetilde{G}$ orbits which contain at least one regular element of $\mathcal{G}$ is Zariski open and dense in $\mathcal{G}$. This can be reformulated as there exist a set $\mathcal{X}$ with the following two properties: (1) $\mathcal{G} \backslash \mathcal{X}$ is a Zariski dense open subset of $\mathcal{G}$ and (2) for each $g \in \mathcal{G} \backslash \mathcal{X}$ there is a regular $d$ and an outer automorphism of $\mathcal{U}_{\xi}$ inducing an isomorphism of algebras $\mathcal{U}_{\xi} / I_{g} \rightarrow \mathcal{U}_{\xi} / I_{d}$. Therefore, for each $g \in \mathcal{G} \backslash \mathcal{X}$ the algebra $\mathcal{U}_{\xi} / I_{g}$ is semi-simple. By replacing $\mathcal{X}$ with $\mathcal{X} \cup \mathcal{X}^{-1}$ we can assume $\mathcal{X}$ is symmetric. Thus, we have shown $\mathscr{C}_{\text {odd }}$ is a generically finitely $\mathcal{G}$-semi-simple category with singular locus $\mathcal{X}$.

Remark 17. By definition, every $\widetilde{G}$ orbit in $\mathcal{G}$ that contains an element of $\mathcal{G} \backslash \mathcal{X}$ also contains a regular element and thus a diagonal element of $\mathcal{G}$.

Recall $\mathscr{C}_{\text {odd }}^{H}$ is graded by the group $\mathcal{D}$. Let $\mathcal{X}_{\mathcal{D}}$ be all the non-regular elements of $\mathcal{D}$. It follows from Lemma 7.1 of [8] that $\mathscr{C}_{\text {odd }}^{H}$ is a generically $\mathcal{D}$-semi-simple category with the singular locus $\mathcal{X}_{\mathcal{D}}$

The results described here and in Section 3 imply the following proposition.

Proposition 18. The category $\mathscr{C}_{\text {odd }}^{H}$ is a generically $\mathcal{D}$-semi-simple braided pivotal category.

4.4. The ribbon structure in $\mathscr{C}_{\text {odd }}^{H}$. Let $V$ be a generic simple object of $\mathscr{C}_{\text {odd }}^{H}$. We will show that $\theta_{V^{*}}=\left(\theta_{V}\right)^{*}$. Since $V$ is simple, the morphism $\theta_{V}$ is a scalar times the identity of $V$. Moreover, $\left(\theta_{V}\right)^{*}$ is the same scalar times $\operatorname{Id}_{V^{*}}$. The module $V$ is determined by its highest weight which is of the form $\lambda+(r-1) \rho$. Its dual $V^{*}$ has highest weight $-\lambda+(r-1) \rho$. To compute the twist on $V$ notice that $H H^{h}$ is the only part of the R-matrix contributing to the following computation:

$$
\theta_{V}(v)=\operatorname{ptr}_{R}\left(c_{V, V}\right)(v)=q^{\langle\lambda, \lambda\rangle-(r-1)^{2}\langle\rho, \rho\rangle} v
$$

where $v$ is a highest weight vector of $V$ (see [19] for more details). As $\langle-\lambda,-\lambda\rangle=\langle\lambda, \lambda\rangle$ then a similar calculation shows that the scalar determining $\theta_{V^{*}}$ is equal to the scalar determining both $\theta_{V}$ and $\left(\theta_{V}\right)^{*}$. Thus, Proposition 18 and the results of this subsection imply that $\mathscr{C}_{\text {odd }}^{H}$ satisfies the hypothesis of Theorem 9 and so we have:

Theorem 19. The category $\mathscr{C}_{\text {odd }}^{H}$ is a ribbon category.

Note, in [19] we showed that a subcategory of $\mathscr{C}_{\text {odd }}^{H}$ is ribbon.

4.5. The trace on the ideal of projective $\mathcal{U}_{\xi}$-modules. From Theorem 4.7.1 of [18] the ideal Proj of projective $\mathcal{U}_{\xi}$-modules in $\mathscr{C}_{\text {odd }}$ admits a unique nontrivial right trace which we denote by

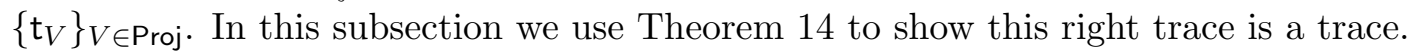

To apply the theorem we need to show $\mathrm{d}(V)=\mathrm{d}\left(V^{*}\right)$ for any generic simple $\mathcal{U}_{\xi}$-module $V$. We now explain how we reduce this equality to a computation related to open Hopf links. Let $V_{0}$ be the weight $\mathcal{U}_{\xi}$-module determined by the highest weight vector $v_{0}$ such that $X_{i} v_{0}=0$ for 
$i=1, \ldots, n$ and $K_{\gamma} v_{0}=q^{\sum_{i} d_{i} c_{i}\left\langle(r-1) \rho, \alpha_{i}\right\rangle} v_{0}$ for $\gamma=\sum_{i} c_{i} \alpha_{i} \in L$. The module $V_{0}$ is isomorphic to $V_{0}^{*}$. To simplify notation, in the following computations we identify these modules with an isomorphism. Given a simple module and a morphism $g: W \rightarrow W$ define $\langle g\rangle \in \mathbb{C}$ as $g=\langle g\rangle \operatorname{Id}_{W}$. Suppose $f: V_{0} \otimes V \rightarrow V_{0} \otimes V$ is a morphism in $\mathscr{C}$. Then

$$
\mathrm{d}(V)\left\langle\operatorname{ptr}_{L}(f)\right\rangle=\mathrm{t}_{V}\left(\operatorname{ptr}_{L}(f)\right)=\mathrm{t}_{V_{0}^{*}}\left(\left(\operatorname{ptr}_{R}(f)\right)^{*}\right)=\mathrm{d}\left(V_{0}^{*}\right)\left\langle\left(\operatorname{ptr}_{R}(f)\right)^{*}\right\rangle=\mathrm{d}\left(V_{0}\right)\left\langle\operatorname{ptr}_{R}(f)\right\rangle
$$

where the second equality is from Lemma 4(b) of [22] and the final equality holds because $V_{0}$ is isomorphic to $V_{0}^{*}$. Therefore, to compute $\mathrm{d}(V)$ we need to give a morphism $f: V_{0} \otimes V \rightarrow V_{0} \otimes V$ and compute its left and right partial trace. We will do this now using the braiding of $U_{h}(\mathfrak{g})$.

Recall the algebra $U_{h}$ and its R-matrix given in Subsection 4.2. Let $V_{0}^{h}$ be the simple $U_{h}$-weight module with highest weight $(r-1) \rho$. So $V_{0}^{h}$ has dimension $r^{N}$ and a highest weight vector $v_{0}$ such that $H_{i} v_{0}=\left\langle(r-1) \rho, \alpha_{i}\right\rangle v_{0}$ for $i=1, \ldots, n$. The module $V_{0}^{h} \otimes \mathbb{C}\left[h^{-1}\right]$ contains its $\mathcal{U}_{q}$ analog $V_{0}^{q}=\mathcal{U}_{q} \cdot v_{0}$ which specialize at $q=\xi$ to the $\mathcal{U}_{\xi}$-module $V_{0}$.

We consider the $U_{h}$-module $V_{0}^{h} \otimes U_{h}$. Let $W_{0} \subset L_{R}$ be the set of weights of $V_{0}^{h}$ and $p^{\lambda}$ the projector of $V_{0}^{h}$ on the weight space of weight $\lambda$. In general, if $v$ and $w$ are vectors of weight $\lambda$ and $\mu$, respectively then $H H^{h}(v \otimes w)=q^{\langle\lambda, \mu\rangle} v \otimes w=v \otimes K_{\lambda} w$. So the action of $H H^{h}$ on $V_{0}^{h} \otimes U_{h}$ is given by

$$
H H^{h}=\sum_{\lambda \in W_{0}} p^{\lambda} \otimes K_{\lambda}
$$

Consider the endomorphism $f_{U_{h}}$ of $V_{0}^{h} \otimes U_{h}$ given by the square of the braiding, that is $f_{U_{h}}$ is the multiplication by $R_{21} R_{12}$. Since $X_{ \pm \alpha}^{r}$ vanishes on $V_{0}^{h}$ the only portion of the quasi R-matrix contributing to $f_{U_{h}}$ is formed by terms of the truncated quasi R-matrix. Equations (8) and (10) imply $f_{U_{h}}$ defines an element $f_{\mathcal{U}_{q}} \in \operatorname{Aut}_{\mathbb{K}_{\ell}}\left(V_{0}^{q}\right) \otimes \mathcal{U}_{q}$. Moreover, these equation imply that $q$ can be specialized at $\xi$ to obtain an element $f_{\mathcal{U}_{\xi}} \in \operatorname{Aut}_{\mathbb{C}}\left(V_{0}\right) \otimes \mathcal{U}_{\xi}$. Then for any representation $\rho_{V}: \mathcal{U}_{\xi} \rightarrow \operatorname{End}(V), f_{V}=\left(\operatorname{Id} \otimes \rho_{V}\right)\left(f_{\mathcal{U}_{\xi}}\right)$ is an automorphism of the $\mathcal{U}_{\xi}$-module $V_{0} \otimes V\left(f_{*}\right.$ defines a natural automorphism of the functor $\left.V_{0} \otimes *\right)$.

Lemma 20. For any generic simple $\mathcal{U}_{\xi}$-module $V$ we have $\operatorname{ptr}_{R}\left(f_{V}\right)=r^{N} \operatorname{Id}_{V_{0}}$.

Proof. Consider a highest weight vector $v_{0}$ of $V_{0}$. Then Equation (10) implies that

$$
\check{R}^{<}\left(v_{0} \otimes x\right)=v_{0} \otimes K_{2(r-1) \rho} x+\left(\text { terms } \in V_{0}^{\prime} \otimes \mathcal{U}_{\xi}\right)
$$

where $x \in \mathcal{U}_{\xi}$ and $V_{0}^{\prime}$ is the sum of weight spaces of $V_{0}$ of weight strictly less than $(r-1) \rho$. Therefore,

$$
\operatorname{ptr}_{R}\left(f_{V}\right)\left(v_{0}\right)=\operatorname{ptr}_{R}\left(p^{(r-1) \rho} \otimes \rho_{V}\left(K_{2(r-1) \rho}\right)\right)\left(v_{0}\right)=v_{0}\left(\sum_{i} x_{i}^{*}\left(K_{2 \rho}^{1-r} K_{2(r-1) \rho} x_{i}\right)\right)=r^{N} v_{0}
$$

where $x_{i}$ is a basis of $V$ and $x_{i}^{*}$ is its dual basis. Since $V_{0}$ is $\operatorname{simple}$ then $\operatorname{ptr}_{R}\left(f_{V}\right)=r^{N} \operatorname{Id}_{V}$.

Let $V$ be a simple module of $\mathscr{C}_{g}$ where $g \in \mathcal{G} \backslash \mathcal{X}$. Then there exists an outer automorphism $\gamma \in \widetilde{G}$ such that $\rho_{V} \circ \gamma$ is a nilpotent representation of $\mathcal{U}_{\xi}$ (see Remark 17). We say $\rho_{V} \circ \gamma$ is a nilpotent deformation of $V$.

Lemma 21. The element $\delta_{q}=\operatorname{ptr}_{L}\left(f_{\mathcal{U}_{q}}\right)$ belongs to the center of $\mathcal{U}_{q}$ and can be specialized to obtain an element $\delta_{\xi}$ in the center of $\mathcal{U}_{\xi}$. Then for any generic simple $\mathcal{U}_{\xi}$-module $V$ we have 
$\operatorname{ptr}_{L}\left(f_{V}\right)=\rho_{V}\left(\delta_{\xi}\right)$. Moreover

$$
\operatorname{ptr}_{L}\left(f_{V}\right)=\prod_{\alpha \in \Delta^{+}} \frac{\{r\langle\mu, \alpha\rangle\}}{\{\langle\mu, \alpha\rangle\}} \operatorname{Id}_{V}
$$

where $\mu+(r-1) \rho$ is the highest weight of a nilpotent deformation of $V$.

Proof. Recall the morphism $f_{U_{h}}: V_{0}^{h} \otimes U_{h} \rightarrow V_{0}^{h} \otimes U_{h}$ is given by multiplication on the left by a truncated part of $R_{21} R_{12}$. Using Equations (8) and (10) a direct computation shows that $\operatorname{ptr}_{L}\left(f_{U_{h}}\right)$ is a $U_{h}$-endomorphism given by left multiplication of an element $\delta_{h}$ of $U_{h}$. Thus, $\delta_{h}$ is an element of the center of $U_{h}$. Moreover, the direct calculation above shows $\delta_{h}$ is a element of $\mathcal{U}_{q}$ seen as a sub algebra of $U_{h}\left[h^{-1}\right]$. By definition this element is equal to $\delta_{q}=\operatorname{ptr}_{L}\left(f_{\mathcal{U}_{q}}\right)$. Therefore, $\delta_{q}$ is central since $\delta_{h}$ is central.

Let $Z_{\xi}$ be the specialization at $q=\xi$ of the center of $\mathcal{U}_{q}$. Then by definition of the derivations $\underline{e}_{i}$ and $\underline{f}_{i}$ it follows that the automorphisms of $\widetilde{G}$ acts trivially on $Z_{\xi}$. Since $\delta_{\xi} \in Z_{\xi}$, it acts as the same scalar endomorphism on $V$ and on any of its nilpotent deformation. Hence the general formula in Equation (11) can be deduce from the case when $V$ is the nilpotent module with highest weight $\mu+(r-1) \rho$ which was computed in Proposition 45 of [19].

Theorem 22. The right trace $\left\{\mathrm{t}_{V}\right\}_{V \in \operatorname{Proj}}$ on Proj in $\mathscr{C}_{\text {odd }}$ is a trace. This trace is unique up to a global scalar since this is true for the right trace.

Proof. Let $V$ be a generic simple $\mathcal{U}_{\xi}$-module. Combining Equation (9) and Lemmas 20 and 21 we have

$$
\mathrm{d}(V)=\frac{\mathrm{d}\left(V_{0}\right)\left\langle\operatorname{ptr}_{R}(f)\right\rangle}{\left\langle\operatorname{ptr}_{L}(f)\right\rangle}=\mathrm{d}\left(V_{0}\right) r^{N} \prod_{\alpha \in \Delta^{+}} \frac{\{\langle\mu, \alpha\rangle\}}{\{r\langle\mu, \alpha\rangle\}}
$$

where $\mu+(r-1) \rho$ is the highest weight of a nilpotent deformation of $V$. The dual of such a nilpotent module has highest weight $-\mu+(r-1) \rho$. Thus, Equation 12 implies $\mathrm{d}(V)=\mathrm{d}\left(V^{*}\right)$. Now from Subsections 3.2 and 4.3 , $\mathscr{C}_{\text {odd }}$ is a generically $\mathcal{G}$-semi-simple pivotal category so the theorem follows from Theorem 14 .

Corollary 23. There exists a unique trace (up to global scalar) on the projective modules of $\overline{\mathscr{C}}_{\text {odd }}$.

Proof. Consider the projection morphism $\mathcal{U}_{\xi} \rightarrow \overline{\mathcal{U}}_{\xi}$. Using this morphism each projective $\overline{\mathcal{U}}_{\xi}$ weight module becomes a projective $\mathcal{U}_{\xi}$ weight module. In fact we have $\overline{\mathscr{C}}_{\text {odd }} \cong \bigoplus_{g \in \mathcal{G}_{0}} \mathscr{C}_{g}$ where $\mathcal{G}_{0}=\left\{g \in \mathcal{G}: g\left(X_{ \pm \beta}^{r}\right)=0, g\left(K_{\beta}^{r}\right)= \pm 1, \beta \in \Delta_{+}\right\}$.

It follows that the trace of Theorem 22 induces a trace on the idea of projective $\overline{\mathcal{U}}_{\xi}$-module. This trace is unique because from Theorem 4.7.1. of [18] there exists unique nontrivial right trace on the projective ideal of $\overline{\mathcal{U}}_{\xi}$-modules.

\section{THE CASE OF $\mathfrak{s l}(2)$}

In the previous subsections we considered three versions of the quantum groups associated to any Lie algebra $\mathfrak{g}$, associated to different lattices $L$ when $q$ was a root of unity of odd order. The main reason the we do not treat even ordered roots of unity above is because we use results on the quantum coadjoint action which at this point require odd order roots of unity (see [11]). In this section we treat the case of all orders of roots of unity for the Drinfeld-Jimbo quantization of $\mathfrak{s l}(2)$ (i.e. the root lattice $L_{R}$ ). In this case we can prove the needed results involving the quantum coadjoint action directly using the casimir element. 
Let $\ell \geq 2, \xi=\mathrm{e}^{2 i \pi / \ell}$ and $r=\frac{2 \ell}{3+(-1)^{\ell}}$. As above we consider the three versions of quantum $\mathfrak{s l}(2)$ associated with the root lattice:

$$
\begin{gathered}
\mathcal{U}_{\xi}(\mathfrak{s l}(2))=\mathcal{U}=\left\langle E, F, K^{ \pm 1} \mid K E-\xi^{2} E K=F K-\xi^{2} K F=[E, F]-\frac{K-K^{-1}}{\xi-\xi^{-1}}=0\right\rangle, \\
\mathcal{U}_{\xi}^{H}(\mathfrak{s l}(2))=\mathcal{U}^{H}=\langle\mathcal{U}, H \mid[H, K]=[H, E]-2 E=[H, F]+2 F=0\rangle \quad \text { and } \\
\overline{\mathcal{U}}=\mathcal{U} /\left(E^{r}, F^{r}, K^{2 r}-1\right) .
\end{gathered}
$$

As above, let $\mathscr{C}_{\mathfrak{s l}(2)}, \mathscr{C}_{\mathfrak{s l}(2)}^{H}$ and $\overline{\mathscr{C}}_{\mathfrak{s l}(2)}$ be their categories of weight modules, respectively. These categories are graded as follows: Let $Z_{0}$ be the subalgebra generated by $E^{r}, F^{r}$ and $K^{r}$. The center of $\mathcal{U}$ is generated by $Z_{0}$ with the casimir

$$
\Omega=\{1\}^{2} E F+K \xi^{-1}+K^{-1} \xi=\{1\}^{2} F E+K \xi+K^{-1} \xi^{-1}
$$

which satisfies the polynomial equation $\mathrm{C}_{r}(\Omega)=\{1\}^{2 r} E^{r} F^{r}-(-1)^{\ell}\left(K^{r}+K^{-r}\right)$ where $\mathrm{C}_{r}$ is the renormalized $r^{t h}$ Chebyshev polynomial (determined by $\mathrm{C}_{r}(2 \cos \theta)=2 \cos (r \theta)$ ).

The set

$$
\mathcal{G}=\left\{M(\kappa, \varepsilon, \varphi)=\left(\left(\begin{array}{ll}
1 & \varepsilon \\
0 & \kappa
\end{array}\right),\left(\begin{array}{cc}
\kappa & 0 \\
\varphi & 1
\end{array}\right)\right): \varepsilon, \varphi \in \mathbb{C}, \kappa \in \mathbb{C}^{*}\right\}
$$

is a group where the multiplication is given by component wise matrix multiplication. Also, as above $\operatorname{Hom}_{\text {alg }}\left(Z_{0}, \mathbb{C}\right)$ is a group where the multiplication is given by $g_{1} g_{2}=g_{1} \otimes g_{2} \circ \Delta$. Then the morphism $\operatorname{Hom}_{\text {alg }}\left(Z_{0}, \mathbb{C}\right) \rightarrow \mathcal{G}$ given by

$$
M(\kappa, \varepsilon, \varphi)\left(K^{r}\right)=\kappa, \quad M(\kappa, \varepsilon, \varphi)\left(E^{r}\right)=\{1\}^{-r} \varepsilon \quad \text { and } \quad M(\kappa, \varepsilon, \varphi)\left(F^{r}\right)=(-1)^{\ell}\{1\}^{-r} \varphi \kappa^{-1} .
$$

is a group homomorphism. We use this morphism to identify $\mathcal{G}$ and $\operatorname{Hom}_{\text {alg }}\left(Z_{0}, \mathbb{C}\right)$.

For each $g \in \mathcal{G}$ let $\mathscr{C}_{g}$ be the full subcategory of $\mathscr{C}_{\mathfrak{s l}(2)}$ whose objects are modules where each $z \in Z_{0}$ act by $g(z)$. As above, for $g \in \mathcal{G}$ with $g\left(E^{r}\right)=g\left(F^{r}\right)=0$ we say $g$ is diagonal and the modules of $\mathscr{C}_{g}$ are nilpotent. Also, a diagonal element $g$ is regular if $g\left(K^{r}\right) \neq \pm 1$. The category $\mathscr{C}_{\mathfrak{s l}(2)}^{H}$ is graded by the group $\mathcal{D} \simeq(\mathbb{C} / 2 \mathbb{Z},+) \simeq \mathfrak{h}^{*} / L_{R} \simeq\left(\mathbb{C}^{*}, \times\right)$ of diagonal elements of $\mathcal{G}$. Finally $\overline{\mathscr{C}}_{\mathfrak{s l}(2)}$ is $\mathbb{Z} / 2 \mathbb{Z}$-graded as a full subcategory of $\mathscr{C}_{\mathfrak{s l}(2)}$.

As above it is known that the element $\phi=K_{2 \rho}^{1-r}$ in $\mathcal{U}, \mathcal{U}^{H}$ and $\overline{\mathcal{U}}$ satisfies $S^{2}(x)=\phi x \phi^{-1}$. It follows that $\mathscr{C}_{\mathfrak{s l}(2)}, \mathscr{C}_{\mathfrak{s l}(2)}^{H}$ and $\overline{\mathscr{C}}_{\mathfrak{s l}(2)}$ are all pivotal $\mathbb{C}$-categories. Moreover, $\mathcal{U}^{H}$ is braided, see [19] for the odd case and [20] for the even.

Lemma 24. The category $\mathscr{C}_{\mathfrak{s l}(2)}$ is generically $\mathcal{G}$-semi-simple category with singular locus

$$
\mathcal{X}=\left\{M(\kappa, \varepsilon, \varphi): \kappa+\frac{1}{\kappa}-\frac{\varepsilon \varphi}{\kappa}= \pm 2\right\} .
$$

Proof. A direct calculations shows $\mathcal{X}$ is small and symmetric. We consider the quantum coadjoint action $\widetilde{G}$ on $\mathcal{G}$, see [10] and above. Let $B \subset G$ be the union of all $\widetilde{G}$-orbits which contain a regular element. To prove the lemma we will show that the category $\mathscr{C}_{h}$ is semi-simple for each $h \in B$ and that $\mathcal{G} \backslash \mathcal{X} \subset B$. To do this we follow an argument similar to the one in Subsection 4.3 direct construction shows that for any regular $g$ the category $\mathscr{C}_{g}$ has $r$ non-isomorphic highest weight modules of dimension $r$ (also see Corollary 3.2 of [10]). Thus, the PBW Theorem and Corollary 7 imply that for regular $g$, the category $\mathscr{C}_{g}$ semi-simple.

Now let $h=M\left(\kappa_{h}, \varepsilon, \varphi\right) \in B$ then by definition of $B$ there exists regular $g=M\left(\kappa_{g}, 0,0\right) \in \mathcal{G}$ such that $g$ and $h$ are in the same $\widetilde{G}$-orbit. Then the $\widetilde{G}$-action gives an outer automorphism of $\mathcal{U}$ inducing an isomorphism of algebras $\mathcal{U} / I_{g} \rightarrow \mathcal{U} / I_{h}$ and so $\mathscr{C}_{h}$ is semi-simple. Finally, the 
element $\Omega_{q}=\{1\}^{2} E F+K q^{-1}+K^{-1} q$ is in the center of the $\mathbb{K}_{\ell-\text { algebra }} \mathcal{U}_{q}$ and specializes to $\Omega$. The quantum coadjoint action is constant on $\Omega=\Omega_{\xi}$ because as explained above this action is constant on the sub-algebra of $\mathcal{U}$ corresponding to the center of $\mathcal{U}_{q}$. Therefore, if $V$ and $W$ are modules of $\mathscr{C}_{g}$ and $\mathscr{C}_{h}$, respectively then $\mathrm{C}_{r}(\Omega)$ acts on both $V$ and $W$ by

$$
-(-1)^{\ell}\left(\kappa_{g}+\kappa_{g}^{-1}\right)=(-1)^{\ell} \varepsilon \varphi \kappa_{h}^{-1}-(-1)^{\ell}\left(\kappa_{h}+\kappa_{h}^{-1}\right) .
$$

Since $g$ is regular then $\kappa_{g} \neq \pm 1$, so $-\varepsilon \varphi \kappa_{h}^{-1}+\kappa_{h}+\kappa_{h}^{-1} \neq \pm 2$. Thus, $h \in \mathcal{G} \backslash \mathcal{X}$.

Corollary 25. The category $\mathscr{C}_{\mathfrak{s}(2)}^{H}$ is ribbon.

Proof. Let $\mathcal{X}_{H}=\mathbb{Z} / 2 \mathbb{Z} \subset \mathbb{C} / 2 \mathbb{Z}$ and let $d \in \mathbb{C} / 2 \mathbb{Z} \backslash \mathcal{X}_{H}$. The proof of Lemma 24 and the forgetful functor $\mathscr{C}_{\mathfrak{s l}(2)}^{H} \rightarrow \mathscr{C}_{\mathfrak{s l}(2)}$ imply $\mathscr{C}_{d}$ has $r$ non-isomorphic highest weight modules of dimension $r$ (one can also see this by direct construction). Then the PBW Theorem and Corollary 7 imply that $\mathscr{C}_{d}$ is semi-simple. Combining this with the results at the beginning of this subsection we have $\mathscr{C}_{\mathfrak{s l}(2)}^{H}$ is generically $\mathcal{G}$-semi-simple pivotal braided category with singular locus $\mathcal{X}_{H}$. It is known that for a generic simple object $V$ that $\theta(V)^{*}=\theta\left(V^{*}\right)$, see [19] for the odd case and [20] for the even. The corollary follows from Theorem 9 .

Note when $\ell$ is ever Corollary 25 was previously known, see [25, 26, 20]. When $\ell$ is odd it was known that $\mathscr{C}_{\mathfrak{s l}(2)}^{H}$ contained a subcategory which is ribbon, see [19].

Next we prove that $\mathscr{C}_{\mathfrak{s l}(2)}$ has a trace and show the modified dimensions only depend on the action of the casimir element. First, the projective cover of the trivial module is self dual, see [15, 16] for the even case and [18] for the odd. Therefore, Corollary 3.2.1 of [18] implies the ideal Proj of projective $\mathcal{U}_{\xi}$-modules admits a unique (up to global scalar) nontrivial right trace which we denote by $\left\{\mathrm{t}_{V}\right\}_{V \in \text { Proj. }}$. We will show this right trace is a trace. As above let $U_{h}=U_{h}(\mathfrak{s l}(2))$ be the h-adic quantum group generated by $E, F$ and $H$. Let $V_{0}^{h}$ be the highest weight module of $U_{h}$ with a highest weight vector $v_{0}$ such that $H v_{0}=(r-1) v_{0}$. Let $V_{0}^{q}$ be the highest weight $\mathcal{U}_{q}$-module with a highest weight vector $v$ such that $K v_{0}=q^{r-1} v_{0}$. Let $V_{0}$ be the highest weight $\mathcal{U}$-module which is the specialization of $V_{0}^{q}$. Let $W_{0}$ be the set of weights of $V_{0}^{h}$. Then $W_{0} \subset L_{R}$ if $\ell$ is odd and $W_{0} \subset \frac{1}{2} L_{R}$ if $\ell$ is even. Let $f_{U_{h}}$ and $f_{\mathcal{U}_{q}}$ be as in Subsection 4.5. As above in the odd case $q$ can be specialized at $\xi$ to obtain an element $f_{\mathcal{U}_{\xi}} \in \operatorname{Aut}_{\mathbb{C}}\left(V_{0}\right) \otimes \mathcal{U}$ and any for representation $\rho_{V}: \mathcal{U} \rightarrow \operatorname{End}(V), f_{V}=\left(\operatorname{Id} \otimes \rho_{V}\right)\left(f_{\mathcal{U}_{\xi}}\right)$ is an automorphism of the $\mathcal{U}$-module $V_{0} \otimes V$.

Lemma 26. For any generic simple $\mathcal{U}_{\xi}(\mathfrak{s l}(2))$-module $V$ we have $\operatorname{ptr}_{R}\left(f_{V}\right)=r \operatorname{Id}_{V_{0}}$.

Proof. The proof of Lemma 20 applies here for general $\ell$.

Lemma 27. There exists a polynomial $P(X) \in \mathbb{C}[X]$ such that for any generic simple $\mathcal{U}_{\xi}(\mathfrak{s l}(2))$ module $V$ we have $\operatorname{ptr}_{L}\left(f_{V}\right)=P(\omega) \operatorname{Id}_{V}$ where $\omega=\left\langle\rho_{V}(\Omega)\right\rangle$.

Proof. As in the proof of Lemma 21 the morphism $\operatorname{ptr}_{L}\left(f_{U_{h}}\right)$ is given by left multiplication of a central element $\delta_{h}$ of $U_{h}(\mathfrak{s l}(2))$. The center of $U_{h}(\mathfrak{s l}(2))$ is generated by the casimir element $\Omega_{h}$. So there exists a polynomial $P(X) \in \mathbb{C}[X]$ such that $\delta_{h}=P\left(\Omega_{h}\right)$. Here the partial closure is taken in $U_{h}(\mathfrak{s l}(2))$. On the other hand, the partial trace of the central element $\delta_{q}=\operatorname{ptr}_{L}\left(f_{\mathcal{U}_{q}}\right)$ is taken in $\mathcal{U}_{q}(\mathfrak{s l}(2))$-mod. Recall that the pivotal structure of $U_{h}(\mathfrak{s l}(2))$-mod and $\mathcal{U}_{q}(\mathfrak{s l}(2))$-mod differ by $K^{-r}$, see Remark 15. In particular, taking the left closer with respect to $V_{0}^{h}$ and $V_{0}^{q}$ differ by the constant $(-1)^{r-1}$ determined by $K^{-r} v_{0}=q^{-r(r-1)} v_{0}=(-1)^{r-1} v_{0}$. Therefore, $\delta_{h}$ viewed as an element of $\mathcal{U}_{q}$ (i.e. seen as the sub algebra of $U_{h}\left[h^{-1}\right]$ ) is equal to the element $(-1)^{r-1} \delta_{q}$. Now 
the morphism $\operatorname{ptr}_{L}\left(f_{V}\right)$ is given by left multiplication by $\delta_{\xi}$ which is specialization a of $\delta_{q}$. Thus, $\operatorname{ptr}_{L}\left(f_{V}\right)=(-1)^{r-1} P(\omega) \operatorname{Id}_{V}$ where $\omega=\left\langle\rho_{V}(\Omega)\right\rangle$.

Corollary 28. Let $V$ be a generic simple $\mathcal{U}_{\xi}(\mathfrak{s l}(2))$-module. Then there exists $\alpha \in(\mathbb{C} \backslash \mathbb{Z}) \cup r \mathbb{Z}$ such that $\left\langle\rho_{V}(\Omega)\right\rangle=(-1)^{r}\left(q^{\alpha}+q^{-\alpha}\right)$ and

$$
\mathrm{d}(V)=(-1)^{r-1} \prod_{j=1}^{r-1} \frac{\{j\}}{\{\alpha+r-j\}}=(-1)^{r-1} \frac{r\{\alpha\}}{\{r \alpha\}}=\frac{(-1)^{r-1} r}{q^{(1-r) \alpha}+\cdots+q^{(r-3) \alpha}+q^{(r-1) \alpha}} .
$$

Moreover, $\mathrm{d}(V)=\mathrm{d}\left(V^{*}\right)$. Here we have fixed the global scalar of the right trace by defining $\mathrm{d}\left(V_{0}\right)=\mathrm{t}_{V_{0}}\left(\operatorname{Id}_{V_{0}}\right)=(-1)^{r-1}$.

Proof. From Equation (9) we have $\mathrm{d}(V)=\frac{\mathrm{d}\left(V_{0}\right)\left\langle\operatorname{ptr}_{R}(f)\right\rangle}{\left\langle\operatorname{ptr}_{L}(f)\right\rangle}$. So by Lemmas 26 and 27 we have $\mathrm{d}(V)=\mathrm{d}(W)$ for any generic simple $W$ such that $\left\langle\rho_{W}(\Omega)\right\rangle=\left\langle\rho_{V}(\Omega)\right\rangle$. The proof of Lemma 24 implies there exists a nilpotent simple module $W$ in the quantum co-adjoint orbit of $V$. Moreover, this proof implies that the action of $\Omega$ is the same on $V$ and $W$. Since $W$ is nilpotent there exists $\alpha \in(\mathbb{C} \backslash \mathbb{Z}) \cup r \mathbb{Z}$ such that $\Omega$ acts on $W$ by $(-1)^{r}\left(q^{\alpha}+q^{-\alpha}\right)$. From [9] it is shown that the modified quantum dimension of $W$ is given by the formula in Equation (13). Thus, Equation (13) follows.

To see the last statement of the corollary, notice $S(\Omega)=\Omega$ so $\left\langle\rho_{V}(\Omega)\right\rangle=\left\langle\rho_{V^{*}}(\Omega)\right\rangle$ and $\mathrm{d}(V)=$ $\mathrm{d}\left(V^{*}\right)$.

Theorem 14, Lemma 24 and Corollary 28 imply the following corollary.

Corollary 29. The right trace $\left\{\mathrm{t}_{V}\right\}_{V \in \operatorname{Proj}}$ on Proj in $\mathscr{C}_{\mathfrak{s l}(2)}$ is a trace.

Corollary 30. There exists a unique (up to global scalar) trace on the projective modules of $\overline{\mathscr{C}}_{\mathfrak{s l}(2)}$.

\section{Conjectures for quantum groups at even ordered roots of unity}

Let $\mathfrak{g}$ be a simple finite-dimensional complex Lie algebra with data $\mathfrak{h}, \Delta^{+}, L_{R}, L_{W}, \ldots$ discussed in Subsection 3.1. Let $\ell$ be an even integer such that $\ell \geq 2$ (and $\ell \notin 3 \mathbb{Z}$ if $\mathfrak{g}=G_{2}$ ). Let $\xi=\mathrm{e}^{2 \sqrt{-1} \pi / \ell}$ and $r=\frac{\ell}{2}$. As above, for each lattice $L$, consider the three versions of the quantum groups: $\mathcal{U}_{\xi}^{L}(\mathfrak{g}), \mathcal{U}_{\xi}^{H}(\mathfrak{g})$ and $\overline{\mathcal{U}}_{\xi}^{L}(\mathfrak{g})$.

The case when $\ell$ is even the situation is not very well developed at this time and needs additional work to apply the results of this paper. For example, the subalgebra $Z_{0}$ of $\mathcal{U}_{\xi}^{L}(\mathfrak{g})$ generated by $\left\{X_{ \pm \beta}^{r}, K_{\gamma}^{r}: \beta \in \Delta_{+}, \gamma \in L\right\}$ is not necessarily commutative: if $\left\langle\beta, \alpha_{i}\right\rangle=1$ then Equation (4) implies

$$
K_{\beta}^{r} X_{j}=q^{r\left\langle\beta, \alpha_{i}\right\rangle} X_{j} K_{\beta}^{r}=-X_{j} K_{\beta}^{r} .
$$

So one must first define an appropriate notion of weight modules for the algebras $\mathcal{U}_{\xi}^{L}(\mathfrak{g}), \mathcal{U}_{\xi}^{H}(\mathfrak{g})$ and $\overline{\mathcal{U}}_{\xi}^{L}(\mathfrak{g})$. Also, as mentioned above the quantum co-adjoint action is not worked out for case when $\ell$ is even. Some work in this direction has been done for $\ell \in 4 \mathbb{Z}$, see [2].

We are lead to the following question, "For even ordered $\xi$, can one define categories of weight modules $\mathscr{C}_{\text {even }}, \mathscr{C}_{\text {even }}^{H}$ and $\overline{\mathscr{C}}_{\text {even }}$ over $\mathcal{U}_{\xi}^{L}(\mathfrak{g}), \mathcal{U}_{\xi}^{H}(\mathfrak{g})$ and $\overline{\mathcal{U}}_{\xi}^{L}(\mathfrak{g})$, respectively such that the following conjectures are true?"

Conjecture 31. For even ordered $\xi$, there exists a category $\mathscr{C}_{\text {even }}^{H}$ of modules over $\mathcal{U}_{\xi}^{H}(\mathfrak{g})$ which is ribbon. 
Conjecture 32. For even ordered $\xi$, there exists a category $\mathscr{C}_{\text {even }}$ of weight modules over $\mathcal{U}_{\xi}^{L}(\mathfrak{g})$ such that there exists a unique (up to global scalar) two sided trace on the ideal of projective modules of $\mathscr{C}_{\text {even }}$.

Conjecture 33. For even ordered $\xi$, there exists a category $\overline{\mathscr{C}}_{\text {even }}$ of modules over $\overline{\mathcal{U}}_{\xi}^{L}(\mathfrak{g})$ such that there exists a unique (up to global scalar) two sided trace on the ideal of projective modules of $\overline{\mathscr{C}}_{\text {even }}$.

The conjectures in this section are motivated by applications in low-dimensional topology. In particular, when $\ell=4$ and $\mathfrak{g}=\mathfrak{s l}(2)$ in [5] it is shown that the closed 3-manifold invariant of [8] associated to the category $\mathscr{C}_{\mathfrak{s l}(2)}^{H}$ are a canonical normalization of Reidemeister torsion defined by Turaev which gives rise to a Topological Quantum Field Theory (TQFT). It would be interesting to see what properties the analogous topological invariants have for other Lie algebras at similar level. The first step in defining such invariants is a proof of Conjecture 31. Also, Conjecture 33 would also be interesting in generalizing the work of [3].

\section{REFERENCES}

[1] J. Barrett, B. Westbury - Spherical categories, Adv. Math. 143 (1999), 357-375.

[2] J. Beck - Representations of quantum groups at even roots of unity J. Algebra 167 (1994), no. 1, 29-56.

[3] A. Beliakova, C. Blanchet, N. Geer - Logarithmic Henning invariants for restricted quantum sl(2), in preparation.

[4] J. Bichon - Cosovereign Hopf algebras, J. Pure Appl. Algebra 157, No.2-3 (2001), 121-133.

[5] Blanchet, C., Costantino, F., Geer, N., and Patureau-Mirand, B., Non-semi-simple TQFTs, Reidemeister torsion and Kashaev's invariants. Advances in Mathematics 301 (2016), 1-78.

[6] C. Blanchet, N. Geer, B. Patureau-Mirand, N. Reshetikhin, - Holonomic ribbon categories and their link invariants, in preparation.

[7] V. Chari, A. Pressley, A guide to quantum groups, Cambridge University Press, Cambridge, 1994.

[8] F. Costantino, N. Geer, B. Patureau-Mirand - Quantum invariants of 3-manifolds via link surgery presentations and non-semi-simple categories, Journal of Topology (2014) 7 (4) 1005-1053.

[9] F. Costantino, N. Geer, B. Patureau-Mirand - Some remarks on the unrolled quantum group of sl(2),, J. Pure Appl. Algebra 219 (2015), no. 8, 3238-3262.

[10] C. De Concini, V.G. Kac - Representations of quantum groups at roots of 1 . In Operator algebras, unitary representations, enveloping algebras, and invariant theory. (Paris, 1989), 471-506, Progr. Math., 92, Birkhauser Boston, 1990.

[11] C. De Concini, V.G. Kac, C. Procesi - Quantum coadjoint action. J. Amer. Math. Soc. 5 (1992), no. 1, $151-189$.

[12] C. De Concini, V.G. Kac, C. Procesi - Some remarkable degenerations of quantum groups. Comm. Math. Phys. 157 (1993), no. 2, 405-427.

[13] C. De Concini, C. Procesi, N. Reshetikhin, M. Rosso - Hopf algebras with trace and representations. Invent. Math. 161 (2005), no. 1, 1-44.

[14] P. Etingof, O. Golberg, S. Hensel, T. Liu, A. Schwendner, D. Vaintrob, E. Yudovina,- Introduction to representation theory, Stud. Math. Libr., 59, Amer. Math. Soc., Providence, RI, 2011.

[15] B.L. Feı̆gin, A.M.Gaŭnutdinov, A.M. Semikhatov, I.Y. Tipunin - Modular group representations and fusion in logarithmic conformal field theories and in the quantum group center. Comm. Math. Phys. 06 (2006); 265 (1), 47-93.

[16] B.L. Fĕgin, A.M.Gaĭnutdinov, A.M. Semikhatov, I.Y. Tipunin - Kazhdan-Lusztig correspondence for the representation category of the triplet W-algebra in logarithmic CFT, Theor Math Phys (2006) 148 (3), $1210-1235$.

[17] N. Geer, J. Kujawa, B. Patureau-Mirand - Generalized trace and modified dimension functions on ribbon categories Selecta Mathematica, New Series 17, Issue 2 (2011) 453-504.

[18] N. Geer, J. Kujawa, B. Patureau-Mirand - Ambidextrous objects and trace functions for nonsemisimple categories, Proceedings of the American Mathematical Society, 141 (2013), no. 9, 2963-2978.

[19] N. Geer, B. Patureau-Mirand - Topological invariants from non-restricted quantum groups. Algebraic \& Geometric Topology 13 (2013) 3305-3363.

[20] N. Geer, B. Patureau-Mirand, V. Turaev - Modified quantum dimensions and re-normalized link invariants. Compos. Math. 145 (2009), no. 1, 196-212. 
[21] N. Geer, B. Patureau-Mirand, V. Turaev - Modified 6j-Symbols and 3-manifold invariants. Advances in Mathematics Volume 228, Issue 2, (2011), 1163-1202.

[22] N. Geer, B. Patureau-Mirand, A. Virelizier, - Traces on ideals in pivotal categories. Quantum Topology, 4 (2013), no. 1, 91-124.

[23] N.P. Ha - Topological invariants from quantum group $U_{q} \mathfrak{s l}(2 \mid 1)$ at root of unity preprint (2016), arXiv:0903.4512.

[24] S. Majid - Foundations Of Quantum Group Theory, Cambridge, UK. Univ. Pr. (1995).

[25] J. Murakami - Colored Alexander invariants and cone-manifolds, Osaka J. Math. 45 (2008), 541-564.

[26] T. Ohtsuki - Quantum invariants. A study of knots, 3-manifolds, and their sets. Series on Knots and Everything, 29. World Scientific Publishing Co., Inc., River Edge, NJ, 2002.

[27] V.G. Turaev - Quantum invariants of knots and 3-manifolds. de Gruyter Studies in Mathematics, 18. Walter de Gruyter \& Co., Berlin, (1994).

Mathematics \& Statistics, Utah State University, Logan, Utah 84322, USA

E-mail address: nathan.geer@gmail.com

Univ. Bretagne - Sud, UMR 6205, LMBA, F-56000 Vannes, France

E-mail address: bertrand.patureau@univ-ubs.fr 